\title{
Can cluster merger shocks reproduce the luminosity and shape distribution of radio relics?
}

\author{
Sebastián E. Nuza ${ }^{1,2,3 \star}$, Jakob Gelszinnis ${ }^{4}$, Matthias Hoeft ${ }^{4}$, and Gustavo Yepes ${ }^{5}$ \\ ${ }^{1}$ Leibniz-Institut für Astrophysik Potsdam, An der Sternwarte 16, 14482 Potsdam, Germany \\ ${ }^{2}$ Universidad de Buenos Aires, Facultad de Ciencias Exactas y Naturales, Buenos Aires, Argentina \\ ${ }^{3}$ CONICET-Universidad de Buenos Aires, Instituto de Astronomía y Física del Espacio (IAFE), Buenos Aires, Argentina \\ ${ }^{4}$ Thüringer Landessternwarte, Sternwarte 5, 07778 Tautenburg, Germany \\ ${ }^{5}$ Grupo de Astrofísica, Universidad Autónoma de Madrid, Cantoblanco, 28039 Madrid, Spain
}

\begin{abstract}
Radio relics in galaxy clusters are believed to trace merger shock fronts. If cosmological structure formation determines the luminosity, size and shape distributions of radio relics then merger shocks need to be lighted up in a homogeneous way. We investigate if a mock relic sample, obtained from zoomed galaxy cluster simulations, is able to match the properties of relics measured in the NRAO VLA Sky Survey (NVSS). We compile a list of all radio relics known to date and homogeneously measure their parameters in all NVSS images and apply the same procedure to relics in our simulations. Number counts in the mock relic sample increase more steeply towards lower relic flux densities, suggesting an incompleteness of NVSS in this regime. Overall, we find that NVSS and mock samples show similar properties. However, large simulated relics tend to be somewhat smaller and closer to the cluster centre than observed ones. Besides this, the mock sample reproduces very well-known correlations for radio relics, in particular those relating the radio luminosity with the largest linear size and the Xray luminosity. We show that these correlations are largely governed by the sensitivity of the NVSS observations. Mock relics show a similar orientation with respect to the direction to the cluster centre as the NVSS sample. Moreover, we find that their maximum radio luminosity roughly correlates with cluster mass, although displaying a large scatter. The overall good agreement between NVSS and the mock sample suggests that properties of radio relics are indeed governed by merger shock fronts, emitting in a homogeneous fashion. Our study demonstrates that the combination of mock observations and data from upcoming radio surveys will allow to shed light on both the origin of radio relics and the nature of the intracluster medium.
\end{abstract}

Key words: shock waves - radiation mechanisms: non-thermal - methods: numerical - galaxies: clusters: general - large-scale structure of Universe

\section{INTRODUCTION}

Diffuse extended radio sources in galaxy clusters with a steep power-law spectrum and without optical counterparts are known since many decades (Feretti \& Giovannini 1996). The sources found in the periphery of clusters were initially attributed to radio galaxies in an inactive phase, i.e. relics of radio galaxies. In such a scenario, the radio spectrum should show a clear break towards high frequencies as a result of the ageing of the electron population. Instead, relics show spectra close to a power-law at least up to frequencies of about $10 \mathrm{GHz}$, suggesting the existence of recently accelerated electrons. Ensslin et al. (1998) proposed a link between

* E-mail: snuza@aip.de radio relics and the shock fronts induced by galaxy cluster mergers. The study of several spectacular relics seems to support this scenario, as suggested by the following facts: (i) X-ray surface brightness jumps attributed to shock fronts have been found in these systems; (ii) spectral index maps of the diffuse radio emission indicate that electron populations are ageing towards the cluster centre (i.e., opposite to the propagation direction of the shock), and (iii) relics display a significant degree of polarization with B-vectors aligned with the shock surface, as can be seen, for instance, in the relic in Abell 3667 (Rottgering et al. 1997, Finoguenov et al. 2010 ), in the 'Sausage' relic in CIZA J2242 (van Weeren et al. 2010. Stroe et al. 2013, Akamatsu et al. 2015), and in the 'Toothbrush' relic in 1RXS J0603 (van Weeren et al. 2012 2016).

(C) 0000 RAS 
Systematic searches for relics have been carried out, e.g. by investigating both X-ray bright Abell-type clusters Giovannini et al. 1999) in the NRAO VLA Sky Survey (NVSS; Condon et al. 1998) and Abell clusters (Kempner \& Sarazin 2001) accessible to the Westerbork Northern Sky Survey (WENSS; Rengelink et al. 1997), by searching for steep spectrum sources in the NVSS (van Weeren et al. 2011), or as a by-product- in the Giant Metrewave Radio Telescope (GMRT) radio halo survey, which is based on a redshiftand flux-limited cluster sample selected from the REFLEX and eBCS catalogues (Kale et al. 2015). Recently, the signature of the Sunyaev-Zel'dovich (SZ) effect in the intracluster medium (ICM) has also been used to identify galaxy clusters (see e.g., Planck Collaboration et al. 2015) that can be later chosen as targets for the relic searches (Bagchi et al. 2011. de Gasperin et al. 2015). All of these works, with the exception of the search performed by van Weeren et al. (2011), have been done by observing at the location of rather massive galaxy clusters. Therefore, it is not surprising that only very few relic candidates are known to be present in poor clusters.

The sample of known radio relics shows a plethora of correlations between relic and cluster properties. Some of them include: the relation between radio emission and cluster X-ray luminosity or mass (Feretti et al. 2012; de Gasperin et al. 2014), between radio relic luminosity and redshift (de Gasperin et al. 2014) and largest linear size (LLS; Feretti et al. 2012). Moreover, radio relics tend to be tangentially oriented with respect to the isocontours of the X-ray surface brightness distribution of clusters (van Weeren et al. 2011). Despite of the existence of these intriguing correlations, their origin, and the systematic effects shaping them, are up to now little studied.

As aforementioned, radio relics most likely originate from cluster merger shock fronts. For several relics, the jump in X-ray surface brightness and/or temperature maps has been clearly measured (e.g., Sarazin et al. 2014, Akamatsu et al. 2015, van Weeren et al. 2016; Botteon et al. 2016). We note, however, that the Mach numbers inferred from X-ray jumps tend to be lower than those expected from the spectral slope of radio observations. In addition to the shocks found in several clusters, the disturbance of the cluster Xray surface brightness, as measured by power ratios, centroid shifts and concentration parameters, shows that relics are, in general, related to ongoing mergers (Yuan et al. 2015).

The high temperatures of the ICM result from shock dissipation, hence, the latter must be included when simulating its evolution. In a pioneering work, Miniati et al. (2000) studied the properties of shock fronts in a cosmological simulation. These authors distinguished between accretion shocks, responsible of heating the primordial intergalactic medium, and merger shocks, which show a complex three-dimensional structure and provide additional gas heating. The properties and distribution of cosmological shock waves have been studied in several subsequent works (e.g., Ryu et al. 2003, Pfrommer et al. 2006 Skillman et al. 2008, Vazza et al. 2009). It has been shown that the distribution and strength of the shocks vary significantly with resolution and numerical scheme (Vazza et al. 2011). However, different simulations basically agree that accretion shocks tend to show Mach numbers of roughly one hundred to one thousand, and that the ICM is filled with a large number of lower Mach number shocks in the range from one to ten. Recent works using improved numerical schemes and increased resolution confirm the abundance and complexity of shock fronts in the ICM (Miniati 2014, Schaal et al. 2016).

It has been known for decades that strong shocks in supernova remnants serve as efficient particle accelerators (see Reynolds 2008 for a review). Even if the physical conditions of intergalactic and intracluster media are quite different from those of the interstellar medium, it appears likely that accretion and merger shocks are also capable to accelerate electrons, protons and nuclei. In this respect, radio relics may be regarded as immediate proof for electron acceleration. However, evidence for proton and nuclei acceleration at these locations is still lacking. A plausible acceleration mechanism is the so-called diffusive shock acceleration (DSA; Drury 1983, Blandford \& Eichler 1987, Caprioli 2012). It has been shown that merger shocks may accelerate electrons and protons via DSA (Kang et al. 2012, Kang \& Ryu 2013). However, a simple scenario for the origin of radio emission in which relativistic electrons originate from the thermal pool by acceleration at the shock front via DSA in the test-particle regime is challenged by several observational findings, namely: (i) Mach numbers derived from the X-ray surface brightness and/or temperature jumps are often too low compared to those inferred from the radio luminosity and spectral index (see e.g., Ogrean et al. 2014, van Weeren et al. 2016); (ii) the spectra of radio relics are possibly curved at frequencies above $10 \mathrm{GHz}$ (Stroe et al. 2016), and (iii) upper limits for the $\gamma$-ray emission from clusters are inconsistent with standard assumptions in DSA (Vazza \& Brüggen 2014. Vazza et al. 2016). Improved modeling including, for instance, re-acceleration of mildly relativistic electrons (Pinzke et al. 2013, Kang \& Ryu 2015, 2016), and/or different acceleration mechanisms such as shock drift acceleration (Guo et al. 2014a), may alleviate the tension between models and observations. We stress that the true origin of the relativistic electrons responsible of the nonthermal radio emission in relics is still a matter of ongoing debate.

The strength, structure and origin of the magnetic fields in relics is also loosely constrained. No direct measurement of their field strength has been possible so far. The most stringent lower limit for the magnitude of the field has been derived for the northern relic in Abell 3667 via upper limits of the inverse Compton (IC) emission, namely $>3 \mu \mathrm{G}$ (Finoguenov et al. 2010). For the relic in the Coma cluster, Bonafede et al. (2013) inferred a field strength of $\sim 3 \mu \mathrm{G}$ using Faraday rotation measurements. The 'Sausage' relic has a small intrinsic width, which suggests a lower limit for the magnetic field strength of $\gtrsim 5 \mu \mathrm{G}$ van Weeren et al. 2010). Magnetic fields in relics may reflect those present in the ICM, however, this underlying field might be also amplified at the shock front, e.g. by the Firehose instability (Guo et al. 2014b), which illustrates the complex non-linear nature of the problem.

For simulating radio relics in cosmological simulations assumptions about both the acceleration of electrons and the magnetic fields in relics have to be made. Hoeft \& Brüggen (2007) introduced a semi-analytic formula relating the nonthermal radio emission, the physical conditions of the downstream medium and the Mach number of the shocks. By grafting this prescription onto simulations it has been shown 

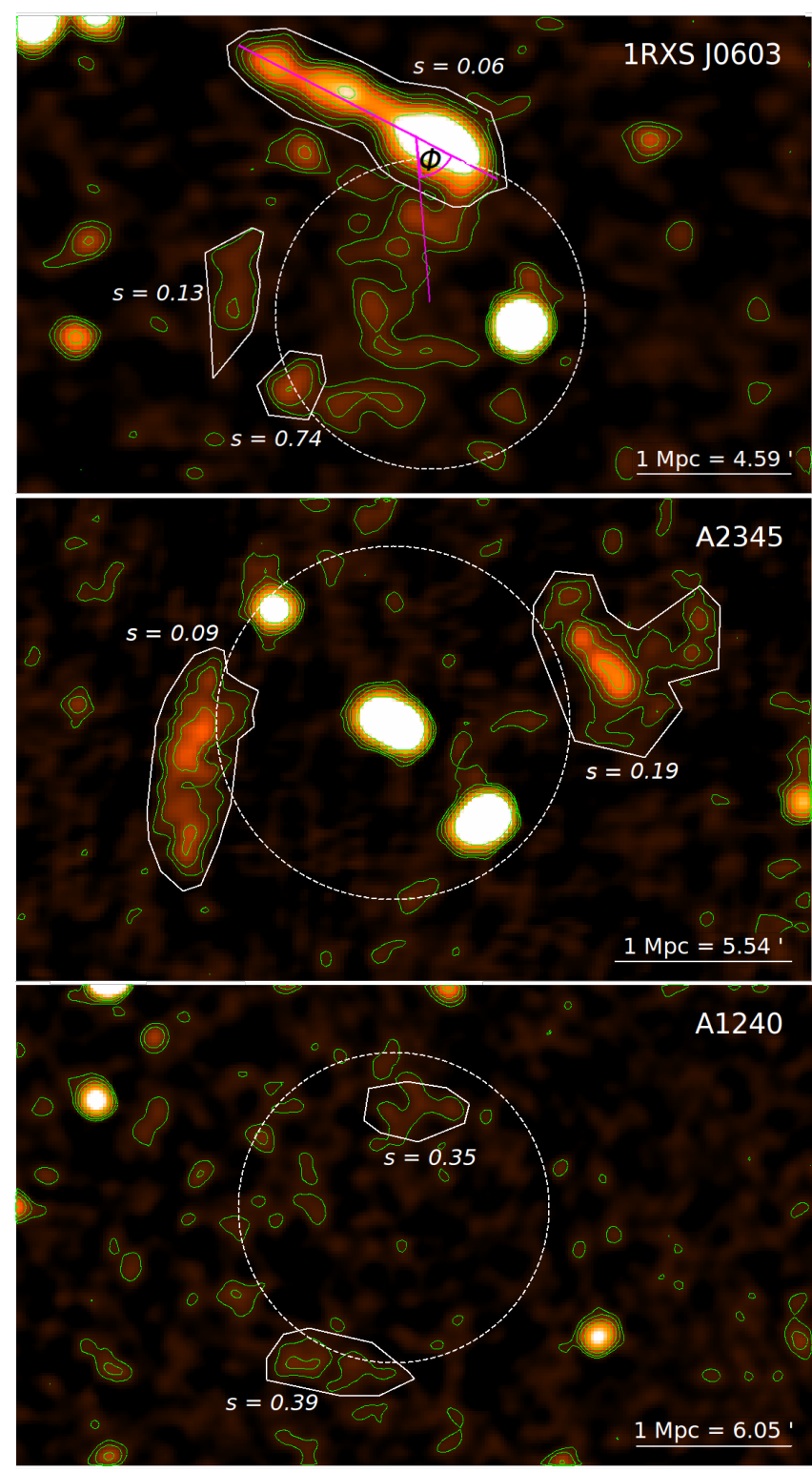

Figure 1. Examples of relic image analysis within NVSS. The colour scale indicates the surface brightness distribution. Contours (green solid lines) are drawn at $[2,4,8,16,32] \times \sigma_{\mathrm{NVSS}}$, where $\sigma_{\text {NVSS }}$ is the average rms brightness fluctuations in the NVSS images. The manually defined relic regions are also shown (white solid lines). Cluster positions are indicated by a circle (white dashed lines) with $1 \mathrm{Mpc}$ radius centred on the coordinates given by SIMBAD. The angle $\phi$ between purple solid lines measures relic orientation. The prominent elongated structure in the top panel is usually referred to as the Toothbrush relic. For Abell 2345 the brightness distribution is shown after subtracting several bright sources in the relic regions.

that cluster mergers may reproduce typical radio relic morphologies (Hoeft et al. 2008, 2011. Skillman et al. 2011). Using higher resolution simulations including the same or similar prescriptions for the radio emission it has been shown that typical relic distances range from a few hundred kpc to about one Mpc from the cluster centre (Vazza et al. 2012) in agreement with observations, and that the spectral index gradient may partly reflect the Mach number distribution of the shock front (Skillman et al. 2013). In contrast, Hong et al. (2015) found that radio emission associated to a set of simulated relic regions was located at distances larger than observed, suggesting that a more efficient electron acceleration at low Mach number shocks could alleviate the discrepancy.

As already mentioned, powerful radio relics are preferentially found in massive galaxy clusters de Gasperin et al. 2014). In order to mimic the entire sample of observed radio relics using simulations, the simulated volume has to be large enough to contain a sufficiently large number of massive clusters. On the other hand, shock fronts need to be resolved with enough resolution to properly describe their morphology. These two conditions can be achieved by means of the so-called 'zooming' technique, where a set of cluster regions, previously selected from a large cosmological box, can be re-simulated at high resolution (Klypin et al. 2001). This technique has been applied to simulate 282 cluster regions drawn from a $1 h^{-3} \mathrm{Gpc}^{3}$ cosmological simulation ( $h$ denoting the reduced Hubble constant) dubbed as the MUSIC-2 galaxy cluster sample (Sembolini et al. 2013).

In a simplified view, merger shock fronts get 'illuminated' via synchrotron emission of relativistic electrons and magnetic fields in the downstream region. To some extent, this resembles the non-related process of stars in galaxies revealing the presence of the dark matter halos hidden in the cosmic web. However, little is known about the relation between radio luminosity and shock properties such as the Mach number. The fact that only about fifty radio relics have been discovered so far may suggests that only the strongest shocks become radio luminous or, alternatively, that additional factors could play a crucial role when forming these objects. In Nuza et al. (2012), to avoid translating cluster merger properties into shock parameters and their corresponding radio luminosities, we derived predictions for the abundance of radio relics simply by introducing the probability of finding radio-luminous shocks with a given power, $P_{1.4}$, as a function of cluster mass.

In this paper we pursue the question if cosmological shock fronts illuminated with a uniform prescription result in a sample of radio relics with a similar flux density distribution, morphology and location within galaxy clusters as the observational one. To this end, we homogeneously measure flux densities, shapes and positions for all known relics and perform a comparison to a mock relic sample. If the properties of the synthetic relics match observations then, any additional requirement for electron acceleration such as, for instance, a pre-existing electron population, must be fulfilled at any other merger shock as well.

The paper is organized as follows. In Section 2 we compile a list of all radio relics known to date and consistently measure their flux densities, shapes, and positions within galaxy clusters found in the NVSS. In Section 3 we describe our mock observations of relics belonging to the simulated MUSIC-2 cluster sample. In Section 4 we perform the comparison between the two samples and, in Section 5, we discuss the results. Finally, in Section 6, we summarize our findings.

Throughout this paper, we assume a $\Lambda$ CDM cosmology with $\Omega_{\mathrm{M}}=0.27, \Omega_{\Lambda}=0.73$ and $H_{0}=70 \mathrm{~km} \mathrm{~s}^{-1} \mathrm{Mpc}^{-1}$. 
Nuza et al.

\section{RADIO RELICS IN THE NVSS}

\subsection{Compilation of known relics to date}

Our aim is to compile a list of radio relics as complete as possible for later comparison with simulations. Feretti et al. (2012) inventoried diffuse radio emission in galaxy clusters and their observational properties. Since then, several new relics have been reported in the literature. In this section, we present an up-to-date collection of relics based on the compilations of Nuza et al. (2012), Feretti et al. (2012), and Yuan et al. (2015), complemented by recently reported objects.

In observations, extended diffuse radio emission without optical counterpart that is located in the cluster periphery is usually classified as a relic. In our simulations, we model radio relics assuming Fermi-I acceleration of electrons at merger shocks. Therefore, the relic sample compiled from observations may systematically differ from the one derived in simulations as the former naturally misses some of the emission which is sufficiently extended or too close to the cluster centre. On the other hand, the observed sample may also comprise diffuse emission without optical counterparts but which does not originate from electron acceleration at merger shocks. Such objects have been identified in observations. A plausible explanation for their origin is given by shock compression of fossil AGN plasma (Enßlin \& GopalKrishna 2001). Following the convention of Kempner et al. (2004), we use the names gischt and phoenix to label diffuse radio emission if it is attributed to Fermi-I acceleration or shock compression, respectively. Since in our simulations we only model radio gischts, a careful inspection of the classification is needed before proceeding with the comparison. In this regard, the spectral properties, and their associated index maps, provide the most clear evidence to perform the classification: an overall power-law spectrum with spectral index steeper than -1 and spectral steeping towards the cluster centre strongly suggest the presence of a gischt (Hoeft \& Brüggen 2007), while a break in the overall spectrum and an irregular distribution of spectral indices indicate that we are dealing with a phoenix.

Interestingly, evidence for a spectral break has also been found for radio gischt textbook examples like the 'Toothbrush' and the 'Sausage' relics (Stroe et al. 2016). However, in these cases, the break occurs at very high frequencies $(\nu>10 \mathrm{GHz})$ and its origin is still debated. In particular, Basu et al. (2016) showed that the SZ effect may have a significant impact at these frequencies.

Most radio relics in our compilation are unambiguously classified either as gischt or phoenix. However, there are some cases where only little information is available and/or the emission properties present contradictory evidence. In those cases, we add the attribute 'candidate' to our classification. Specifically, the latter becomes particularly difficult for diffuse emission which is only moderately extended, located rather close to the cluster centre, and that shows a complex morphology. Three scenarios for the origin of those objects are conceivable: (i) we are in the presence of a radio galaxy which recently went into the passive phase and is significantly separated from the plasma ejecta due to a high velocity w.r.t. the ambient medium; (ii) the plasma has been ejected long time ago and is now re-energised by shock compression, i.e. it is a phoenix, or (iii) it might actually be a small merger shock at an early stage, e.g. see the radio

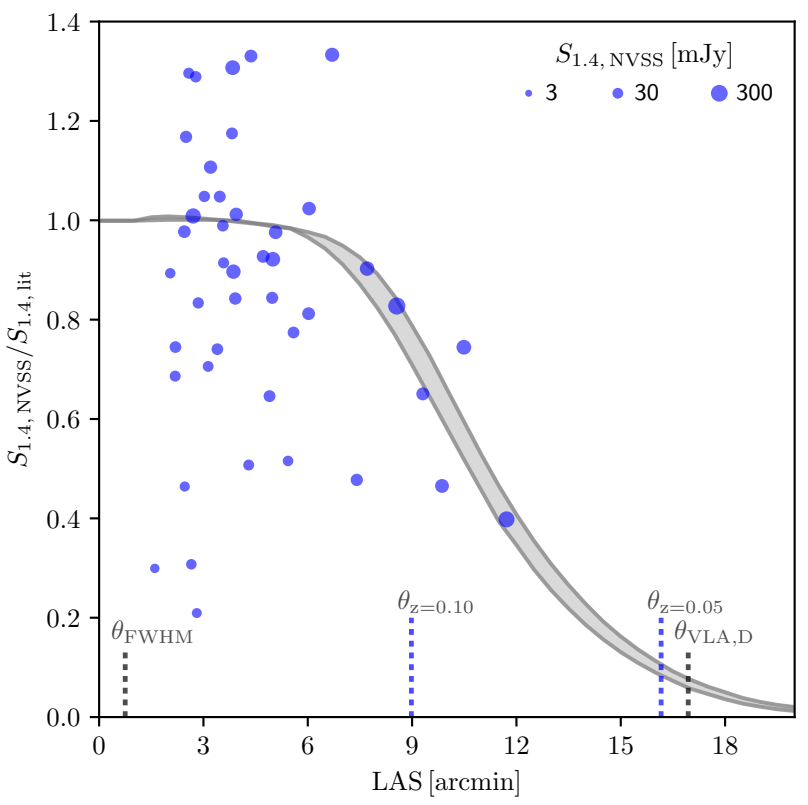

Figure 2. Ratio of flux densities measured in the NVSS images and those reported in literature versus the largest angular size (LAS) of the largest radio-emitting region in each cluster. Symbol size scales with NVSS-measured flux. The shaded grey region shows the expectation for the NVSS flux recovery as derived by Farnsworth et al. (2013). Also shown are relevant angular scales.

ridges in Abell 1351 (Giacintucci et al. 2009) and Abell 1682 (Venturi et al. 2013). To highlight that some cluster mergers may cause small, complex shock fronts giving rise to radio emission according to the gischt scenario, we introduce a new class: the 'gischtlet'. For these objects, several aspects of the standard relic classification do not apply, e.g. they are evidently not located at the cluster outskirts, hence all gischtlets are only 'candidates' in our nomenclature.

We note that, for some objects formerly listed as radio relics, evidence has been found that the emission is actually related to a radio galaxy. For the diffuse emission near the galaxy cluster Abell 786, Kale \& Dwarakanath (2012) reported a compact radio source near the centre of the emission. The diffuse emission near the galaxy cluster Abell 2069 has been also identified to be part of a radio galaxy (Drabent et al. in prep.). Therefore, we do not include these sources in our list.

It has been found that the radio luminosity of relics correlates with X-ray luminosity of the host galaxy cluster. Because of this, we also include cluster X-ray luminosities in the $0.1-2.4 \mathrm{keV}$ energy band in our compilation. For most clusters, we adopted the values given in Feretti et al. (2012). If necessary, we corrected published X-ray luminosities to the cosmology and/or energy band adopted here. For some clusters, where no X-ray luminosity is known but estimates for the virial mass have been published, we adopt the luminosity-to-mass relation given in Section 3.2 to estimate the X-ray emission.

Our compilation contains a total of 69 clusters hosting radio gischt, gischtlet, or phoenix emission including candidates (see Table 11). The majority of the relic-type diffuse 
Table 1. Radio relic collection

\begin{tabular}{|c|c|c|c|c|c|c|c|}
\hline $\begin{array}{l}\text { Cluster } \\
\text { (1) }\end{array}$ & $\begin{array}{l}z \\
(2)\end{array}$ & $\begin{array}{c}L_{\mathrm{X}} \\
{\left[10^{44} \mathrm{erg} \mathrm{s}^{-1}\right]} \\
(3)\end{array}$ & $\begin{array}{c}S_{1.4, \mathrm{lit}} \\
{[\mathrm{mJy}]} \\
(4)\end{array}$ & $\begin{array}{c}S_{1.4, \mathrm{NVSS}} \\
{[\mathrm{mJy}]} \\
(5)\end{array}$ & $\begin{array}{l}\text { Classification } \\
\text { (6) }\end{array}$ & Refe & rences \\
\hline Abell 13 & 0.094 & 1.18 & 31.0 & not gischt & phoenix can. & Fer12 & Sle01 \\
\hline Abell 85 & 0.055 & 4.10 & 43.0 & not gischt & phoenix & Fer12 & Sle01 \\
\hline Abell 115 & 0.197 & 8.91 & 147.0 & 68.4 & gischt & Fer 12 & Gov01 \\
\hline Abell 133 & 0.057 & $1.93^{a}$ & 137.0 & not gischt & phoenix can. & Ebe96 & Ran10 \\
\hline Abell 209 & 0.206 & 6.17 & 1.0 & too faint & gischt can. (+halo) & Gio09 & Gio09 \\
\hline Abell 521 & 0.247 & 8.47 & 15.0 & 13.7 & gischt (+ halo) & Fer12 & Gia06 \\
\hline Abell 523 & 0.104 & 1.07 & 61.0 & 29.1 & gischtlet can. & Fer12 & Wee11a \\
\hline Abell 548b & 0.045 & 0.15 & 121.0 & too extended & gischtlet can. & Fer12 & Fer06 \\
\hline Abell 610 & 0.095 & $0.8^{b}$ & 17.3 & 15.5 & gischt can. & Bos08 & Gio00 \\
\hline Abell 725 & 0.092 & $0.45^{a}$ & 6.0 & not gischt & phoenix can. & Böh00 & Kem01 \\
\hline Abell 746 & 0.232 & 3.68 & 24.5 & 12.4 & gischt (+ halo) & Fer12 & Wee11a \\
\hline Abell S753 & 0.014 & $0.5^{c}$ & 460.0 & not gischt & phoenix can. & Sub03 & Sub03 \\
\hline Abell 754 & 0.054 & 2.21 & 6.0 & too faint & gischtlet can. (+ halo) & Fer12 & Mac11 \\
\hline Abell 781 & 0.300 & 4.6 & 15.5 & 16.2 & gischt $(+$ halo $)$ & Fer 12 & Gov11 \\
\hline Abell 910 & 0.206 & $3.39^{a}$ & 12.1 & contam. & gischtlet can. & Ebe00 & Gov12 \\
\hline Abell 1033 & 0.130 & $4.43^{d}$ & 46.9 & not gischt & phoenix can. & Gas15a & Gas15a \\
\hline Abell 1240 & 0.159 & 1.0 & 16.1 & 11.4 & gischt double & Fer12 & Bon09 \\
\hline Abell 1300 & 0.307 & 13.73 & 20.0 & 19.8 & gischt can. (+ halo $)$ & Fer 12 & Ven 13 \\
\hline Abell 1351 & 0.322 & 8.4 & 13.0 & 16.8 & gischtlet can. (+ halo) & Gia09 & Gia09 \\
\hline Abell 1367 & 0.022 & 0.82 & 232.0 & too extended & gischt can. (+ halo) & Far13 & Far13 \\
\hline Abell 1443 & 0.270 & 6.2 & $9.8^{A}$ & 8.8 & gischt can. (+ halo) & Bon15 & Bon15 \\
\hline Abell 1612 & 0.179 & 2.41 & 62.8 & 61.3 & gischt can. & Fer12 & Wee11a \\
\hline Abell 1656: Coma & 0.023 & 3.99 & 260.0 & too extended & gischt can. (+ halo $)$ & Fer12 & Fer12 \\
\hline Abell 1664 & 0.128 & 3.09 & 107.0 & 96.6 & gischt can. & Fer12 & Gov01 \\
\hline Abell 1682 & 0.226 & 7.0 & $26.9^{B}$ & 20.0 & gischtlet can. (+ halo) & Ven 13 & Ven 13 \\
\hline Abell 1758N & 0.280 & $7.6^{a}$ & 22.1 & 12.9 & gischt double can. (+ halo) & Ebe98 & Gio09 \\
\hline Abell 2034 & 0.113 & 3.90 & 24.0 & 28.0 & gischt can. (+ halo) & Ebe98 & Wee11a \\
\hline Abell 2048 & 0.097 & 1.91 & 19.0 & not gischt & phoenix & Wee11b & Wee11b \\
\hline Abell 2061 & 0.078 & 3.95 & 27.6 & 5.8 & gischt & Wee11a & Wee11a \\
\hline Abell 2063 & 0.035 & 0.98 & $81.5^{C}$ & not gischt & phoenix can. & Fer12 & Kom94 \\
\hline Abell 2163 & 0.203 & 22.73 & 18.7 & 12.8 & gischt can. & Fer12 & Fer01 \\
\hline Abell 2255 & 0.081 & 2.64 & 12.0 & too faint & gischt can. (+ halo) & Fer12 & $\mathrm{Piz08}$ \\
\hline Abell 2256 & 0.058 & 3.75 & 462.0 & 183.9 & gischt can. (+ halo) & $\mathrm{Cla06}$ & $\mathrm{Cla} 06$ \\
\hline Abell 2345 & 0.177 & 5.90 & 59.0 & 87.2 & gischt double & Bon09 & Bon09 \\
\hline Abell 2443 & 0.108 & 1.9 & 6.5 & not gischt & phoenix & Fer12 & Coh11 \\
\hline Abell 2744 & 0.308 & 12.86 & 18.2 & 9.4 & gischt (+ halo) & Fer12 & - Gov01 \\
\hline Abell 3365 & 0.093 & 0.86 & 47.9 & 38.9 & gischt double can. & Wee11a & Wee11a \\
\hline Abell 3376 & 0.046 & 1.08 & 302.0 & too extended & gischt double & Fer 12 & Bag06 \\
\hline Abell 3411 & 0.169 & $53.9^{a}$ & 53.0 & 53.6 & gischt can. (+ halo) & Ebe02 & Wee13 \\
\hline Abell 3527-bis & 0.200 & 1.9 & 35.0 & 22.6 & gischt & Gas 16 & Gas16 \\
\hline Abell 3667 & 0.056 & 4.73 & 2400.0 & low dec. & gischt & Fer12 & Roe97 \\
\hline Abell 4038 & 0.030 & 1.97 & 49.0 & not gischt & phoenix & Fer12 & Sle01 \\
\hline ACT-CL J0102-49: El Gordo & 0.870 & $64.6^{e}$ & $8.6^{D}$ & low dec. & gischt double & $\operatorname{Lin} 14$ & $\operatorname{Lin} 14$ \\
\hline CIZA J0107+54 & 0.107 & 3.9 & 30.0 & 9.2 & gischt double can. & Ran 16 & Ran16 \\
\hline CIZA J0649+18 & 0.064 & 2.38 & $27.5^{E}$ & 21.3 & gischt can. & Wee11a & Raj17 \\
\hline CIZA J2242+53: Sausage & 0.192 & 6.80 & 144.0 & 107.2 & gischt & Fer12 & Str13 \\
\hline CL $0217+70$ & 0.066 & 0.63 & - & contam. & gischt double can. (+ halo) & Fer12 & Bro11 \\
\hline CL $1446+26$ & 0.370 & 3.42 & 5.3 & contam. & gischt can. (+ halo) & Fer12 & Gov12 \\
\hline MACS J0025-1222 & 0.586 & 8.8 & $2.3^{F}$ & too faint & gischt double & Ebe07 & Ris16 \\
\hline MACS J0717+37 & 0.555 & $8.6^{a}$ & 83.0 & 108.5 & gischtlet can. $(+$ halo $)$ & Fer12 & Pan13 \\
\hline MACS J $1149+22$ & 0.544 & 14.0 & 10.3 & 13.3 & gischt double can. & Bon12 & Bon12 \\
\hline MACS J1752+44 & 0.366 & $8.0^{e}$ & $101.8^{G}$ & 91.3 & gischt double (+ halo) & Bon12 & Bon12 \\
\hline MACS J2243-09 & 0.447 & 11.56 & $1.9^{H}$ & too faint & gischt can. & Can 16 & Can16 \\
\hline MaxBCG $138+25$ & 0.324 & - & 24.7 & not gischt & phoenix can. & - & . Wee11b \\
\hline MaxBCG $217+13$ & 0.160 & 1.0 & - & not gischt & phoenix can. & Wee09 & Wee09 \\
\hline PLCK G004-19 & 0.516 & 16.0 & 37.0 & 36.2 & gischt can. & Sif14 & Sif14 \\
\hline PLCK G $287+32$ & 0.390 & 17.2 & 58.0 & 59.4 & gischt double & Fer12 & Bag11 \\
\hline PSZ1 G096+24 & 0.300 & 3.7 & 27.2 & 20.1 & gischt double & Gas14 & Gas14 \\
\hline PSZ1 G108-11 & 0.335 & 7.5 & 113.1 & 104.2 & gischt double (+ halo) & Gas15b & Gas15b \\
\hline RXC J0225-29 & 0.060 & 0.41 & 37.0 & 31.2 & gischt can. & Sha16 & Sha16 \\
\hline RXC J1053+54 & 0.070 & 3.69 & 15.0 & 7.0 & gischt can. & Fer12 & Wee11a \\
\hline $\mathrm{RXC} \mathrm{J1234+09}$ & 0.229 & 6.32 & 3.1 & too faint & gischt can. (+ halo) & Kal15 & Kal15 \\
\hline RXC J1314-25 & 0.247 & 10.75 & 30.3 & 40.3 & gischt doube (+ halo) & Fer05 & Fer05 \\
\hline S1081 & 0.220 & - & 2.4 & too faint & gischt can. & - & Mid08 \\
\hline $\mathrm{ZwCl}_{\mathrm{w}} 0008+52$ & 0.103 & 0.5 & 67.0 & 43.6 & gischt double & Fer12 & . Wee11c \\
\hline $\mathrm{ZwCl} 2341+00$ & 0.270 & 2.4 & 18.5 & 25.2 & gischt double can. & Gas14 & - Gio10 \\
\hline 1E 0657-55: Bullet & 0.296 & 22.59 & 82.6 & low dec. & gischt $(+$ halo $)$ & Gio09 & Shi15 \\
\hline 1RXS J0603+42: Toothbrush & 0.225 & 7.7 & $364.2^{I}$ & 301.2 & gischt & Ogr13 & Wee16 \\
\hline $24 \mathrm{P} 73$ & 0.150 & - & 12.0 & not gischt & phoenix & - & Wee11b \\
\hline
\end{tabular}


Nuza et al.

Table 1 - continued Radio relic collection

Column 1: Cluster name (common names for the cluster or relic are indicated in italic). Column 2: Cluster redshift. Column 3: Cluster X-ray luminosity in the $0.1-2.4 \mathrm{keV}$ energy band: ${ }^{a}$ corrected for cosmology; ${ }^{b}$ estimated via $M_{\mathrm{vir}}=2.05 \times 10^{14} M_{\odot}(\mathrm{Bos} 08)$ and Eq. 7. ${ }^{c}$ estimated via $M_{\text {vir }}=1.5 \times 10^{14} M_{\odot}$ (Sub03) and Eq. $7 .{ }^{d}$ converted to $0.1-2.4 \mathrm{keV}$ band via mekal model with $T=6.39 \mathrm{keV}$ and a metallicity of 0.26 (Gas15a); ${ }^{e}$ converted to $0.1-2.4 \mathrm{keV}$ band via mekal model with $T=14.5 \mathrm{keV}$ (Lin14) and a metallicity of 0.25 . Column 4: Flux density of radio relic as reported in literature: ${ }^{A}$ extrapolated from $323 \mathrm{MHz}$ adopting a spectral index of -1.2 ; ${ }^{B}$ extrapolated from $240 \mathrm{MHz}$ adopting a spectral index of $-1.62 ;{ }^{C}$ mean of the $1.360 \mathrm{GHz}$ and $1.465 \mathrm{GHz}$ flux values; ${ }^{D}$ extrapolated from $610 \mathrm{MHz}$ adopting a spectral index of $-1.2 ;{ }^{E}$ extrapolated from $1.47 \mathrm{GHz}$ adopting a spectral inded of $-1.15 ;{ }^{F}$ extrapolated from $325 \mathrm{MHz}$ adopting a spectral index of $-1.3 ;{ }^{G}$ extrapolated from $1.714 \mathrm{GHz}$ adopting a spectral index of $-1.18 ;{ }^{H}$ extrapolated from $610 \mathrm{MHz}$ adopting a spectral index of $-1.2 ;{ }^{I}$ extrapolated from $1.5 \mathrm{GHz}$ adopting a spectral index of -1.09 ; Column 5: Flux density measured in NVSS images. Relics are excluded when: (i) the origin is most likely not shock acceleration, i.e. not gischt or gischtlet; (ii) the source position is not covered by NVSS, i.e. the declination is too low; (iii) the emission is too faint; (iv) the source is too extended, hence NVSS cannot recover the flux density, and (v) the contamination by other sources is too strong. Column 6: Classification. Column 7: References for cluster X-ray luminosity and radio flux density: Bag06 - Bagchi et al. (2006); Bag11 - Bagchi et al. (2011); Böh00 - Böhringer et al. (2000); Bon09 - Bonafede et al. (2009); Bon12 - Bonafede et al. (2012); Bon15 - Bonafede et al. (2015); Bos08 Boschin et al. (2008); Bro11 - Brown et al. (2011); Can16 - Cantwell et al. (2016); Cla06 - Clarke \& Ensslin (2006); Coh11 - Cohen \& Clarke (2011); Ebe96 - Ebeling et al. (1996); Ebe98 - Ebeling et al. (1998); Ebe00 - Ebeling et al. (2000); Ebe02 - Ebeling et al. (2002); Ebe07 - Ebeling et al. (2007); Far13 - Farnsworth et al. (2013); Fer01 - Feretti et al. (2001); Fer05 - Feretti et al. (2005); Fer06 - Feretti et al. (2006); Fer12 - Feretti et al. (2012); Gas14 - de Gasperin et al. (2014); Gas15a - de Gasperin et al. (2015); Gas15b - de Gasperin et al. (2015); Gas16 - de Gasperin et al. (2016); Gia06 - Giacintucci et al. (2006); Gia09 - Giacintucci et al. (2009); Gio00 - Giovannini \& Feretti (2000); Gio09 - Giovannini et al. (2009); Gio10 - Giovannini et al. (2010); Gov01 - Govoni et al. (2001); Gov11 - Govoni et al. (2011); Gov12 - Govoni et al. (2012); Kal15 - Kale et al. (2015); Kem01 - Kempner \& Sarazin|(2001); Kom94 - Komissarov \& Gubanov (1994); Lin14 - Lindner et al. (2014); Mac11 - Macario et al. (2011); Mid08 - Middelberg et al. (2008); Ogr13 - Ogrean et al. (2013); Pan13 - Pandey-Pommier et al. (2013); Piz08 - Pizzo et al. (2008); Raj17 - Rajpurohit et al. in prep.; Ran10 - Randall et al. (2010); Ran16 - Randall et al. (2016); Ris16 - Riseley et al. (2016); Roe97 - Rottgering et al. (1997); Sif14 - Sifón et al. (2014); Sha16 Shakouri et al. (2016); Shi15 - Shimwell et al. (2015); Sle01 - Slee et al. (2001); Str13 - Stroe et al. (2013); Sub03 - Subrahmanyan et al. (2003); Ven13 - Venturi et al. (2013); Wee09 - van Weeren et al. (2009a); Wee11a - van Weeren et al. (2011); Wee11b - van Weeren et al. (2011); Wee11c - van Weeren et al. (2011); Wee13 - van Weeren et al. (2013); Wee16 - van Weeren et al. (2016).

emission is classified as gischt. In 14 of our clusters, the emission is classified as phoenix, whereas in 6 as gischtlets.

\section{$2.2 \quad$ Analysis of NVSS images}

The relics listed in Table 1 have been studied using different telescopes, observing configurations and/or frequencies. Relics are extended low-surface brightness objects. The deeper the observation is, the more extended faint emission might be revealed. Therefore, properties such as flux density and size reported in literature crucially depend on instrumental parameters like beam size and sensitivity of the observation.

Our aim is to measure relic properties for all known relics in a homogeneous way, even if that implies recovering less flux than reported in literature. The NVSS is well suited for this purpose because it covers about $82 \%$ of the sky and the survey sensitivity is sufficient to recover most of the known relics. The NVSS has been carried out at $1.4 \mathrm{GHz}$, images are made with a Gaussian restoring beam of $\theta_{\mathrm{FWHM}}=45$ arcsec. The root mean square $(\mathrm{rms})$ of the surface brightness fluctuations amounts, in average, to $\sigma_{\text {NVSS }}=0.45$ mJy beam $^{-1}$. We use radio images obtained with the NVSS Postage Stamp Server ${ }^{1}$ and measure flux density, size and shape for the relics listed in Table 1

The measurement of properties for the known relics in a homogeneous fashion is optimally carried out by implementing a semi-automated analysis of the NVSS images. We will later apply the same procedure to analyse our simulated relic sample as it will be discussed in the next section. For every object, we first define a region closely encircling the relics reported in literature. This is done manually in order

1 cv.nrao.edu/nvss/postage.shtml to fully comprise the regions of interest, as well as to exclude compact sources and false positives due to noise. Compact sources within the relic regions have been subtracted from the images using the NVSS restoring beam and adopting flux density values from the high-resolution radio survey FIRST (Becker et al. 1995) or by estimating the flux density in better resolved observations available in the literature. The gischt double relic in Abell 2345 is an example of an object where we have subtracted a bright compact source.

For the threshold encircling the radio emission we adopt the $I_{\text {th }}=2 \times \sigma_{\text {NVSS }}$ contour as the surface brightness limit to define the relic boundaries. We note that there is no danger of detecting a significant amount of spurious emission since the manually defined regions closely encircle the true flux. We have chosen such a low threshold to recover as much emission as possible. Interestingly, even after adopting this value, some relics may decompose into several pieces, e.g. see the diffuse emission in Abell 3411. In what follows, we will denote every radio-emitting piece as an 'island'. Fig. 1 shows three examples of relics in the NVSS where it can be seen that recovered morphologies are quite diverse. The most prominent is the 'Toothbrush' (top panel) which not only excels through its extremely linear, yet non-symmetric appearance, but also through its surface brightness. In fact, the 'Toothbrush' has the highest flux density in our sample. Fainter structures, also reported as relics, are well visible in NVSS within this cluster too.

Obviously, not all relics in our compilation will be accesible to NVSS. In this respect, several reasons can be given: (i) if the object is too far south, resulting in a declination lower than $-40^{\circ}$, it will not be covered; (ii) if the surface brightness is too low the relic may not be detected; (iii) if the relic emission is confused with a bright source the flux and/or the morphological properties will not be easily mea- 
Table 2. Radio relic emission 'islands' identified in the NVSS images.

\begin{tabular}{|c|c|c|c|c|c|c|c|c|c|c|}
\hline $\begin{array}{l}\text { Cluster } \\
\text { (1) }\end{array}$ & $\begin{array}{r}\mathrm{RA} \\
{[\mathrm{deg}]} \\
(2)\end{array}$ & $\begin{array}{r}\text { Dec } \\
{[\mathrm{deg}]} \\
(3)\end{array}$ & $\begin{array}{c}S_{1.4} \\
{[\mathrm{mJy}]} \\
(4)\end{array}$ & $\begin{array}{r}\log _{10}\left(P_{1.4}\right) \\
{\left[\mathrm{W} \mathrm{Hz}^{-1}\right]} \\
(5)\end{array}$ & $\begin{array}{r}\text { LAS } \\
\text { [arcmin] } \\
(6)\end{array}$ & $\begin{array}{c}\text { LLS } \\
{[\mathrm{kpc}]} \\
(7)\end{array}$ & $\begin{array}{c}\Omega \\
{\left[\Omega_{\text {beam }}\right]} \\
(8)\end{array}$ & $\begin{array}{r}\lambda_{2} / \lambda_{1} \\
{[\%]} \\
(9)\end{array}$ & $\begin{array}{r}D_{\text {proj }} \\
{[\mathrm{kpc}]} \\
(10)\end{array}$ & $\begin{array}{r}\phi \\
{[\mathrm{deg}]} \\
(11)\end{array}$ \\
\hline Abell 115 & 14.00 & +26.46 & $68.4 \pm 14.3$ & $24.90_{-0.10}^{+0.08}$ & 10.12 & $1982 \pm 78$ & $20.7 \pm 1.4$ & 8 & 1602 & 64.2 \\
\hline Abell 521 & 73.59 & -10.28 & $13.7 \pm 2.9$ & $24.45_{-0.11}^{+0.10}$ & 3.69 & $874 \pm 95$ & $5.7 \pm 0.8$ & 12 & 895 & 66.0 \\
\hline Abell 523 & 74.79 & +8.81 & $29.1 \pm 8.7$ & $23.91_{-0.15}^{+0.11}$ & 7.63 & $875 \pm 46$ & $16.0 \pm 1.3$ & 39 & 373 & 58.6 \\
\hline Abell 610 & 119.87 & +27.12 & $15.5 \pm 3.2$ & $23.56_{-0.10}^{+0.108}$ & 2.94 & $311 \pm 42$ & $6.2 \pm 0.8$ & 39 & 310 & 88.6 \\
\hline Abell 746 & 137.23 & +51.58 & $12.4 \pm 3.3$ & $24.32_{-0.14}^{+0.10}$ & 4.39 & $977 \pm 89$ & $6.8 \pm 0.8$ & 11 & 1488 & 67.0 \\
\hline Abell 781 & 140.13 & +30.47 & $16.2 \pm 2.7$ & $24.70_{-0.08}^{+0.107}$ & 3.10 & $830 \pm 107$ & $4.8 \pm 0.7$ & 56 & 628 & 61.0 \\
\hline Abell $1240 \mathrm{~N}$ & 170.86 & +43.17 & $6.0 \pm 1.8$ & $23.63_{-0.15}^{+0.08}$ & 3.22 & $531 \pm 66$ & $3.7 \pm 0.6$ & 35 & 642 & 68.8 \\
\hline $\mathrm{S}$ & 170.96 & +43.01 & $5.4 \pm 1.5$ & $23.59_{-0.14}^{+0.10}$ & 2.16 & $356 \pm 66$ & $3.1 \pm 0.6$ & 39 & 1169 & 50.0 \\
\hline Abell 1300 & 172.95 & -19.95 & $19.8 \pm 3.5$ & $24.81_{-0.09}^{+0.07}$ & 3.63 & $987 \pm 109$ & $6.4 \pm 0.8$ & 34 & 1284 & 45.6 \\
\hline Abell 1351 & 175.59 & +58.50 & $16.8 \pm 2.8$ & $24.79_{-0.08}^{+0.07}$ & 2.86 & $805 \pm 112$ & $5.0 \pm 0.7$ & 51 & 713 & 68.8 \\
\hline Abell 1443 & 180.27 & +23.10 & $8.8 \pm 1.6$ & $24.32_{-0.09}^{+0.07}$ & 2.10 & $520 \pm 99$ & $2.9 \pm 0.5$ & 45 & 1268 & 78.0 \\
\hline Abell 1612 & 191.96 & -2.88 & $61.3 \pm 8.6$ & $24.76_{-0.07}^{+0.09}$ & 5.19 & $942 \pm 73$ & $13.4 \pm 1.2$ & 19 & 1071 & 77.1 \\
\hline Abell 1664 & 195.88 & -24.37 & $96.6 \pm 21.2$ & $24.63_{-0.11}^{+0.09}$ & 7.90 & $1084 \pm 55$ & $41.9 \pm 2.0$ & 75 & 1053 & 20.7 \\
\hline Abell 1682-NW & 196.68 & +46.57 & $20.0 \pm 4.1$ & $24.50_{-0.10}^{+0.08}$ & 2.25 & $492 \pm 87$ & $3.5 \pm 0.6$ & 62 & 323 & 27.6 \\
\hline Abell 1758N E & 203.23 & +50.54 & $14.5 \pm 4.9$ & $24.58_{-0.18}^{+0.10}$ & 3.31 & $848 \pm 102$ & $5.7 \pm 0.7$ & 23 & 679 & 61.8 \\
\hline W & 203.16 & +50.56 & $7.6 \pm 1.8$ & $24.30_{-0.12}^{+0.10}$ & 3.21 & $823 \pm 102$ & $3.6 \pm 0.6$ & 20 & 133 & 27.6 \\
\hline Abell $2034 \mathrm{~W}$ & 227.42 & +33.52 & $28.0 \pm 3.6$ & $23.98_{-0.06}^{+0.105}$ & 2.56 & $315 \pm 49$ & $5.0 \pm 0.7$ & 49 & 807 & 54.1 \\
\hline Abell 2061 & 230.00 & +30.53 & $5.8 \pm 1.5$ & $22.95_{-0.13}^{+0.00}$ & 2.88 & $255 \pm 35$ & $3.0 \pm 0.5$ & 19 & 1753 & 82.7 \\
\hline Abell 2163 & 244.06 & -6.09 & $12.8 \pm 2.0$ & $24.20_{-0.08}^{+0.13}$ & 2.25 & $452 \pm 80$ & $3.5 \pm 0.6$ & 43 & 2008 & 26.3 \\
\hline Abell 2256 & 255.85 & +78.73 & $183.9 \pm 29.8$ & $24.18_{-0.08}^{+0.08}$ & 12.07 & $814 \pm 27$ & $52.1 \pm 2.3$ & 19 & 87 & 81.8 \\
\hline Abell $2345 \mathrm{E}$ & 321.90 & -12.19 & $41.6 \pm 8.1$ & $24.58_{-0.10}^{+0.08}$ & 6.90 & $1236 \pm 72$ & $15.3 \pm 1.2$ & 9 & 1173 & 70.9 \\
\hline W & 321.68 & -12.13 & $32.8 \pm 6.1$ & $24.48_{-0.09}^{+0.08}$ & 5.39 & $966 \pm 72$ & $11.9 \pm 1.1$ & 19 & 1227 & 69.6 \\
\hline & 321.63 & -12.12 & $4.3 \pm 1.1$ & $23.59_{-0.13}^{+0.10}$ & 2.33 & $418 \pm 72$ & $2.2 \pm 0.5$ & 14 & 1759 & 62.4 \\
\hline Abell 2744 & 3.67 & -30.35 & $9.4 \pm 2.6$ & $24.49^{+0.11}$ & 5.60 & $1526 \pm 109$ & $5.3 \pm 0.7$ & 9 & 1408 & 74.0 \\
\hline Abell $3365 \mathrm{E}$ & 87.27 & -21.78 & $38.9 \pm 8.3$ & $23.94_{-0.10}^{+0.08}$ & 6.20 & $644 \pm 42$ & $16.2 \pm 1.3$ & 14 & 1620 & 80.0 \\
\hline Abell 3411 a & 130.52 & -17.58 & $27.5 \pm 5.5$ & $24.35_{-0.10}^{+0.08}$ & 4.06 & $703 \pm 69$ & $10.6 \pm 1.0$ & 43 & 1258 & 20.4 \\
\hline $\mathrm{b}$ & 130.55 & -17.63 & $18.3 \pm 3.0$ & $24.18_{-0.08}^{+0.07}$ & 2.65 & $459 \pm 69$ & $5.1 \pm 0.7$ & 35 & 1843 & 12.3 \\
\hline c & 130.56 & -17.54 & $7.8 \pm 1.5$ & $23.80_{-0.09}^{+0.08}$ & 1.97 & $341 \pm 69$ & $2.8 \pm 0.5$ & 41 & 1245 & 84.9 \\
\hline Abell 3527-bis & 192.77 & -37.03 & $22.6 \pm 4.6$ & $24.43_{-0.10}^{+0.09}$ & 5.04 & $1001 \pm 80$ & $8.8 \pm 0.9$ & 8 & 1387 & 86.9 \\
\hline CIZA J0107+54 NE & 16.96 & +54.15 & $4.0 \pm 1.1$ & $23.07_{-0.14}^{+0.10}$ & 2.40 & $282 \pm 47$ & $2.3 \pm 0.5$ & 71 & 77 & 23.4 \\
\hline SW & 16.92 & +54.09 & $5.2 \pm 1.7$ & $23.19^{+0.12}$ & 2.73 & $321 \pm 47$ & $3.7 \pm 0.6$ & 67 & 381 & 68.8 \\
\hline CIZA J0649+18 & 102.16 & +18.02 & $16.4 \pm 3.9$ & $23.22_{-0.12}^{+0.09}$ & 5.72 & $422 \pm 30$ & $7.9 \pm 0.9$ & 14 & 784 & 84.8 \\
\hline & 102.19 & +17.97 & $4.9 \pm 1.0$ & $22.69_{-0.10}^{+0.108}$ & 2.79 & $206 \pm 30$ & $2.0 \pm 0.4$ & 24 & 702 & 60.7 \\
\hline CIZA J2242+53 N & 340.74 & +53.15 & $91.2 \pm 13.0$ & $25.00_{-0.07}^{+0.06}$ & 10.79 & $2072 \pm 77$ & $23.8 \pm 1.5$ & 10 & 1468 & 81.7 \\
\hline & 340.91 & +53.09 & $11.9 \pm 2.2$ & $24.11_{-0.09}^{+0.07}$ & 2.94 & $564 \pm 77$ & $4.5 \pm 0.7$ & 22 & 1550 & 27.5 \\
\hline $\mathrm{S}$ & 340.64 & +52.94 & $4.1 \pm 1.0$ & $23.65_{-0.13}^{+0.09}$ & 2.36 & $454 \pm 77$ & $2.1 \pm 0.5$ & 16 & 1102 & 86.2 \\
\hline MACS J0717+37 & 109.40 & +37.76 & $108.5 \pm 12.0$ & $26.17_{-0.06}^{+0.106}$ & 3.93 & $1524 \pm 155$ & $8.7 \pm 0.9$ & 40 & 20 & 56.6 \\
\hline MACS J1149+22 NW & 177.35 & +22.39 & $7.4 \pm 1.4$ & $24.99_{-0.11}^{+0.08}$ & 2.08 & $797 \pm 154$ & $2.6 \pm 0.5$ & 33 & 1139 & 41.3 \\
\hline $\mathrm{SE}$ & 177.44 & +22.36 & $5.9 \pm 1.4$ & $24.89_{-0.13}^{+0.10}$ & 2.65 & $1019 \pm 154$ & $2.8 \pm 0.5$ & 31 & 1268 & 5.9 \\
\hline MACS J1752+44 NE & 268.09 & +44.70 & $64.2 \pm 7.6$ & $25.50_{-0.06}^{+0.13}$ & 3.95 & $1208 \pm 122$ & $9.0 \pm 0.9$ & 21 & 1093 & 89.6 \\
\hline SW & 267.98 & +44.63 & $27.0 \pm 3.5$ & $25.13_{-0.07}^{+0.06}$ & 3.32 & $1016 \pm 122$ & $5.0 \pm 0.7$ & 45 & 1058 & 70.2 \\
\hline PLCK G004-19 & 289.26 & -33.51 & $36.2 \pm 4.3$ & $25.63_{-0.07}^{+0.06}$ & 2.52 & $954 \pm 151$ & $5.3 \pm 0.7$ & 62 & 274 & 1.2 \\
\hline PLCK G287+32 N & 177.71 & -28.05 & $38.9 \pm 6.9$ & $25.35_{-0.09}^{+0.07}$ & 6.16 & $1961 \pm 127$ & $12.6 \pm 1.1$ & 17 & 517 & 49.6 \\
\hline $\mathrm{S}$ & 177.81 & -28.19 & $20.4 \pm 4.8$ & $25.07_{-0.12}^{+0.09}$ & 5.24 & $1669 \pm 127$ & $9.8 \pm 1.0$ & 16 & 2854 & 71.5 \\
\hline PSZ1 G096+24 N & 284.15 & +66.44 & $7.1 \pm 1.8$ & $24.34_{-0.13}^{+0.10}$ & 2.73 & $731 \pm 107$ & $3.6 \pm 0.6$ & 14 & 985 & 75.4 \\
\hline $\mathrm{S}$ & 284.18 & +66.33 & $13.0 \pm 3.0$ & $24.60_{-0.12}^{+0.13}$ & 3.50 & $938 \pm 107$ & $5.9 \pm 0.8$ & 24 & 850 & 75.3 \\
\hline PSZ1 G108-11 NE & 350.72 & +48.82 & $60.8 \pm 7.6$ & $25.38_{-0.07}^{+0.106}$ & 5.13 & $1471 \pm 115$ & $10.2 \pm 1.0$ & 16 & 1353 & 89.6 \\
\hline SW & 350.56 & +48.66 & $43.4 \pm 6.7$ & $25.23_{-0.08}^{+0.07}$ & 3.80 & $1091 \pm 115$ & $11.4 \pm 1.1$ & 39 & 2069 & 70.9 \\
\hline RXC J0225-29 & 36.47 & -29.61 & $31.2 \pm 4.6$ & $23.45_{-0.07}^{+0.06}$ & 4.03 & $284 \pm 28$ & $7.5 \pm 0.9$ & 20 & 890 & 49.1 \\
\hline RXC J1053+54 & 163.21 & +54.95 & $7.0 \pm 1.5$ & $22.93_{-0.10}^{+0.08}$ & 2.54 & $203 \pm 32$ & $2.8 \pm 0.5$ & 23 & 738 & 88.3 \\
\hline RXC J1314-25 E & 198.70 & -25.26 & $13.4 \pm 2.5$ & $24.42_{-0.09}^{+0.08}$ & 4.36 & $1016 \pm 93$ & $4.8 \pm 0.7$ & 12 & 975 & 69.3 \\
\hline $\mathrm{w}$ & 198.58 & -25.26 & $26.9 \pm 4.6$ & $24.72_{-0.08}^{+0.07}$ & 4.50 & $1049 \pm 93$ & $8.2 \pm 0.9$ & 22 & 483 & 69.1 \\
\hline $\mathrm{ZwCl} 0008+52 \mathrm{E}$ & 3.11 & +52.61 & $33.8 \pm 7.9$ & $23.97_{-0.12}^{+0.09}$ & 9.49 & $1079 \pm 45$ & $16.0 \pm 1.3$ & 4 & 1200 & 87.0 \\
\hline $\mathrm{W}$ & 2.79 & +52.51 & $9.8 \pm 2.7$ & $23.43_{-0.14}^{+0.11}$ & 3.57 & $406 \pm 45$ & $5.7 \pm 0.7$ & 23 & 328 & 85.3 \\
\hline $\mathrm{ZwCl} 2341+00 \mathrm{a}$ & 355.95 & +0.23 & $14.0 \pm 3.5$ & $24.53_{-0.13}^{+0.10}$ & 3.57 & $887 \pm 99$ & $7.2 \pm 0.8$ & 44 & 1293 & 79.8 \\
\hline c & 355.91 & +0.35 & $9.0 \pm 1.9$ & $24.34_{-0.11}^{+0.108}$ & 2.72 & $675 \pm 99$ & $3.7 \pm 0.6$ & 22 & 557 & 72.7 \\
\hline 1RXS J0603+42 S & 90.90 & +42.17 & $6.2 \pm 1.3$ & $23.99_{-0.10}^{+0.11}$ & 1.66 & $362 \pm 87$ & $2.5 \pm 0.5$ & 74 & 1005 & 6.0 \\
\hline SW & 90.94 & +42.22 & $5.7 \pm 1.6$ & $23.96_{-0.14}^{+0.10}$ & 3.01 & $655 \pm 87$ & $3.3 \pm 0.6$ & 13 & 1247 & 89.4 \\
\hline Toothbrush & 90.82 & +42.30 & $289.3 \pm 30.7$ & $25.66_{-0.05}^{+0.04}$ & 8.82 & $1915 \pm 87$ & $22.7 \pm 1.5$ & 6 & 1132 & 58.8 \\
\hline
\end{tabular}

Column 1: Cluster name and island identifier. Columns 2 and 3: Right ascension and declination. Columns 4 and 5: Flux density and rest-frame luminosity at $1.4 \mathrm{GHz}$. Columns 6 to 10: Largest angular size, largest linear size, shape and projected distance to cluster centre. Column 11: Island orientation (see Section 2.2.2. 
sured, and (iv) if the relic is too extended it could not be fully recovered due to missing short spacings in the interferometer. The NVSS was carried out with the VLA in D and DnC configurations, hence, the largest possible angular scale accesible to the survey is $970 \operatorname{arcse}^{2}$ Therefore, for clusters at redhift $z \lesssim 0.05$, relics are too extended for a reliable flux density measurement, i.e. they are "resolved out". We note, however, that even at the largest angular scale configurations, still less than $50 \%$ of the object's flux is collected by the interferometer. As a result, somewhat less extended sources may still not be fully recovered (Farnsworth et al. 2013). In fact, for the extended relics in Abell 2256, Abell 115 and CIZA 2242, we measure less flux in NVSS than reported in the literature (see Fig. 2).

\subsubsection{Flux density, size and area}

For each individual island, we measure the total solid angle, $\Omega$, from the sum of its constituent pixels. We also determine the LAS, $\theta_{\mathrm{LAS}}$, using the smallest enclosing circle. From the latter, we also derive the LLS of the islands adopting our fiducial cosmology. To determine the flux density, $S_{1.4}$, one has to sum the surface brightness over all pixels accross the island and then divide by the beam solid angle. In radioastronomy, the latter is commonly defined as

$$
\Omega_{\text {beam }}=\frac{1}{\ln 2} \Omega_{\mathrm{FWHM}}=\frac{1}{\ln 2} \frac{\pi}{4} \theta_{\mathrm{FWHM}}^{2},
$$

where $\Omega_{\mathrm{FWHM}}$ is the restoring beam solid angle and $\theta_{\mathrm{FWHM}}$ its associated angular scale. For simplicity, we adopt an angular scale of 45 arcsec for the restoring beam width of the survey even if there are small variations across the large sky area covered by NVSS. Since the beam has a Gaussian shape, the maximum flux density of an island can be related to its solid angle by

$$
S_{1.4, \text { max }}(\Omega)=I_{\text {th }} \Omega_{\text {beam }}\left\{\exp \left(\frac{\Omega}{\Omega_{\text {beam }}}\right)-1\right\} .
$$

To avoid including very small islands which might be governed by noise, we discard all objects with a flux density below $S_{1.4, \min }=8 \times \sigma_{\mathrm{NVSS}}$, i.e. $3.6 \mathrm{mJy}$. This value has been chosen to obtain realiable flux measurements while keeping the maximum number of islands possible. From the flux density of each island we also derive its radio luminosity, $P_{1.4}$, assuming an average spectral index of -1.2 for the $k$-correction.

\subsubsection{Shape, position and orientation}

We quantify the shape and orientation of islands via image moments, which are commonly used to characterise images (Stobie 1980 Bertin \& Arnouts 1996). First, we determine the emission-weighted centre, $\left(x_{c}, y_{c}\right)$, of each island. Then, the central moment, $\mu_{p q}$, of order $p+q$ can be computed as

$$
\mu_{p q}=\sum_{(i, j) \in \text { island }}\left(x_{i}-x_{c}\right)^{p}\left(y_{j}-y_{c}\right)^{q} I_{i j}
$$

2 science.nrao.edu/facilities/vla/docs/manuals/oss/performance/ resolution where $\left(x_{i}, y_{j}\right)$ and $I_{i j}$ are the coordinates and surface brightness of the pixels, respectively. We describe the shape $s$ of the relic through the ratio

$$
s \equiv \lambda_{2} / \lambda_{1}
$$

where $\lambda_{1}$ and $\lambda_{2}$ are the first and second eigenvalues of the covariance matrix

$$
\frac{1}{\mu_{00}}\left(\begin{array}{ll}
\mu_{20} & \mu_{11} \\
\mu_{11} & \mu_{02}
\end{array}\right)
$$

As an example, we note that idealized linear and circular objects would have shape parameters of $s=0,1$, respectively. It is evident that the shape of the manually defined relic regions might have some effect on the relic shape. Nevertheless, we checked that the impact of the latter is negligible.

To characterise the location of islands within their host galaxy clusters, we measure their projected distance, $D_{\text {proj }} \equiv\left|\mathbf{r}_{\mathbf{c c}}\right|$, where $\mathbf{r}_{\mathbf{c c}}$ denotes the position vector joining the cluster and island centres. For the cluster centres, we adopt positions from the SIMBAD catalogue (Wenger et al. 2000). We note, however, that these positions are not homogeneously determined, some being derived from the X-ray surface brightness distribution and others via the cluster SZ effect.

Additionally, we determine the orientation of the relic with respect to the cluster. The first eigenvector of the covariance matrix, $\mathbf{v}_{\mathbf{1}}$, is parallel to the major axis of the radio island. Hence, the angle between $\mathbf{v}_{\mathbf{1}}$ and the position vector $\mathbf{r}_{\mathbf{c c}}$, i.e.

$$
\phi=\angle\left(\mathbf{v}_{\mathbf{1}}, \mathbf{r}_{\mathbf{c c}}\right),
$$

indicates how any given island is oriented within its host galaxy cluster, where radial and tangential elongations are given by the angles $\phi=0^{\circ}$ and $90^{\circ}$, respectively (see purple solid lines in Fig. 1).

\subsubsection{Error estimates}

We estimate the uncertainties of island flux densities, luminosities, sizes, projected distances and solid angles. In the case of the flux, we take into account the three dominant error sources contributing to the island flux measurement, namely: (i) the image noise; (ii) the uncertainties caused by point source subtraction, and (iii) the errors in the absolute flux calibration. We simply assume that the flux of the subtracted point sources, $S_{\mathrm{ps}}$, is uncertain by $5 \%$ and that the absolute flux calibration is uncertain by $10 \%$. Since the errors are uncorrelated we estimate the flux density uncertainty as

$$
\Delta S_{1.4}=\sqrt{\left(0.1 S_{1.4}\right)^{2}+\left(0.05 S_{\mathrm{ps}}\right)^{2}+\sigma_{\mathrm{NVSS}}^{2}\left(\frac{\Omega}{\Omega_{\text {beam }}}\right)} .
$$

The uncertainty of the luminosity is obtained by error propagation of the flux density. We assume that measurement of the LAS has an error of about half the beam width, i.e. we take $\Delta \theta_{\mathrm{LAS}}=0.4^{\prime}$. From this uncertainty we compute the corresponding quantity for the LLS. For the projected spatial distance, we simply assume a fiducial error of $100 \mathrm{kpc}$ for all islands. This value is large enough to encompass typical errors resulting from the measurement of the images within the redshift range of the sample. 


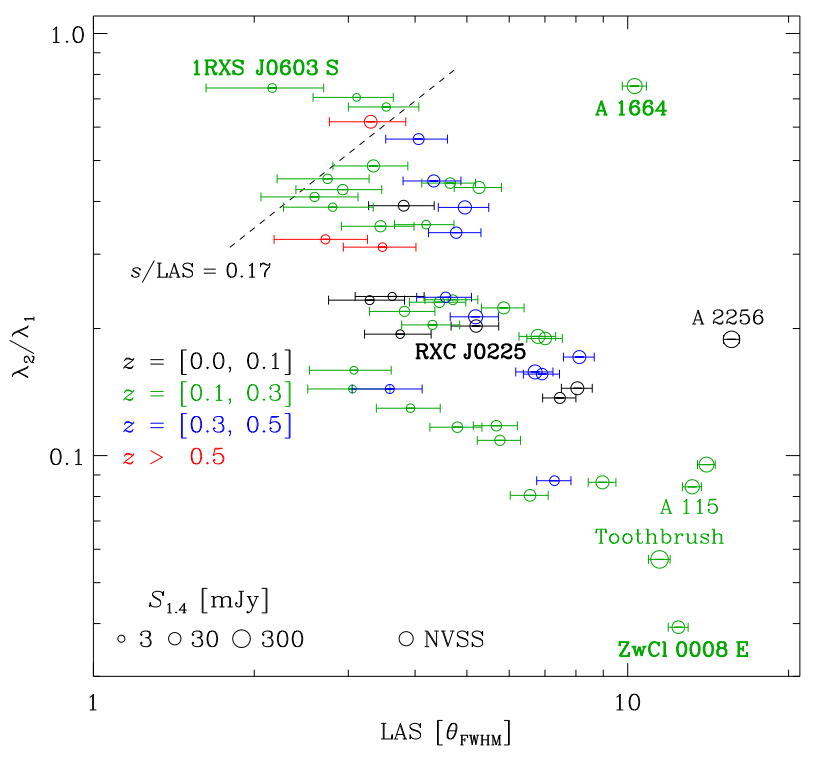

Figure 3. Shape parameter, $s \equiv \lambda_{2} / \lambda_{1}$, versus the largest angular size (LAS) for NVSS relics. Symbol sizes are scaled according to radio flux. Redshift bins are indicated using different colours. The dashed line indicates the empirical relation $s / \mathrm{LAS}=0.17$.

\subsection{Characterising the relic samples}

The measured parameters of all NVSS islands fulfilling our selection criteria are listed in Table 2 To better characterise the relic shape, in Fig. 3 we plot the shape parameter versus the LAS for all NVSS islands (empty circles).

As seen in the plot, the relic shape is anti-correlated with the largest angular size, meaning that, the larger (smaller) the relic is, the more elongated (roundish) results. Interestingly, there are a few outliers to this relation which are clearly not fragments of a larger relic. One quite roundish for its LAS is the gischt candidate in Abell 1664, whose classification is somewhat uncertain. This object shows an extended emission coming from the cluster periphery and no evidence of a spectral break suggesting a gischt interpretation (Kale \& Dwarakanath 2012). However, the cluster's cool core might be considered as an argument against an ongoing merger. Similarly, we also found a somewhat roundish island in Abell 1351. In this cluster the ridge is difficult to disentangle from the halo emission (Giacintucci et al. 2009). Another example is the gischt double relic in MACS J1149, which appears roundish in the NVSS images because the cluster is rather distant and the brightness of the relics is low. The most roundish object in the sample is the southern emission feature in the 'Toothbrush' cluster due to its unusual compactness. For compact emission, the minimum LAS introduced by the surface brightness limit given by Eq. 2 is $\theta_{\text {LAS }}=1.52 \times \theta_{\text {FWHM }}$, which lies below the corresponding angular scale of NVSS islands. A visual impression of the islands can be seen in Fig. 4 where four extreme cases drawn from the shape-LAS correlation are presented.

In general, we find that there are only a few islands with $s / \theta_{\text {LAS }}>0.17$ (see dashed line in Fig. 3). We consider this ratio as an empirical detection limit in NVSS, possibly caused by the fact that point-like sources are usually
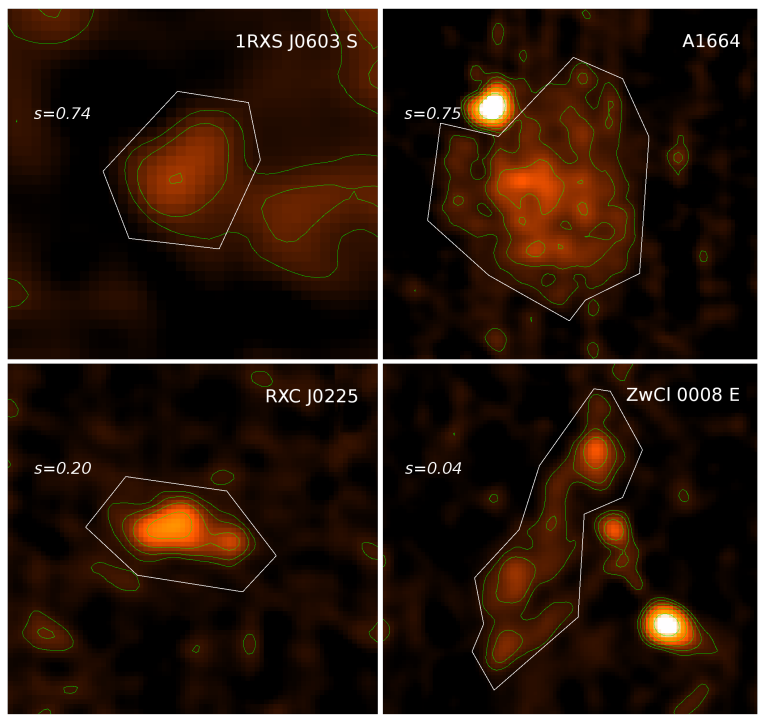

Figure 4. Relic examples drawn from the $s$-LAS correlation (shown in boldface in Fig. 3): 1RXS J0603 S: most roundish and compact object; A1664: largest outlier; RXC J0225: object with median s/LAS, and ZwCl 0008 E: most elongated object. Relic regions are indicated by white solid lines.

not classified as radio relics. For instance, relics in distant clusters may have escaped attention in systematic searches based on NVSS, as they might appear as faint point sources in the radio maps. Therefore, in what follows, we use the ratio $s / \theta_{\mathrm{LAS}}=0.17$ to distinguish between 'small-roundish' and 'elongated' objects. We stress that this distinction will be particularly important when discussing the simulated mock relic sample (see Section 4).

\subsection{NVSS completeness}

Fig. 5 shows the cumulative number of clusters hosting relics versus their total radio flux for the NVSS sample (solid line). For future reference, the cumulative counts of the 'elongated' simulated cluster sample are also shown (shaded regions). These curves represent full-sky extrapolations computed from a set of MUSIC-2 mock cluster samples. A detailed discussion concerning the generation of mock cata$\operatorname{logs}$ and relic abundance is postponed until Sections 4.1 and 4.5 respectively. We note that the total radio flux of all galaxy clusters, both in observations and mocks, is computed adding all diffuse emission coming from their associated islands.

As seen in Fig. 5 , the NVSS curve rapidly flattens below a flux value of about $10 \mathrm{mJy}$. Several effects may contribute to this behaviour. The most obvious is related to detection limit: to be observed, an object's surface brightness has to be larger than the threshold, $I_{\mathrm{th}}$. For instance, a relic covered by ten beams has to have, at least, a flux density larger than $9 \mathrm{mJy}$ to be listed in Table 2. It is also possible that faint relics could reside in low-mass clusters that have not been identified yet. In Nuza et al. (2012) we estimated that about $50 \%$ of relics with a flux density below $10 \mathrm{mJy}$ may reside in clusters below the detection limit of the REFLEX cluster 


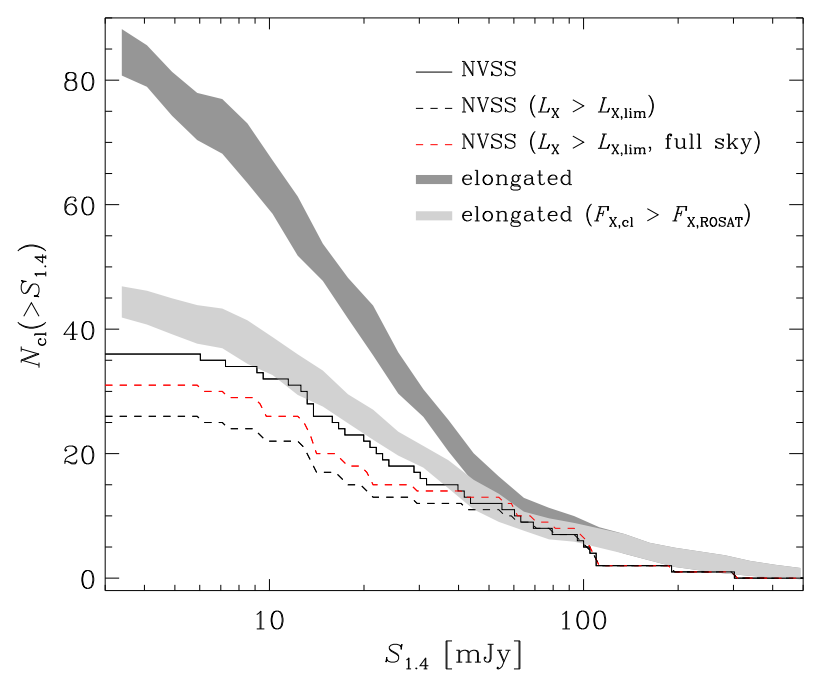

Figure 5. Cumulative number of clusters versus total radio flux density. The cluster flux density is computed adding the contribution of all individual islands obtained from the image analysis described in Section 2.2 Shown are the NVSS (solid line), idem but only for clusters above the X-ray luminosity completeness limit of the MUSIC-2 simulation (dashed line; see Section 3.1), and the latter including a correction for sky incompleteness (red line). Shaded regions indicate the mean and standard deviation for a set of MUSIC-2 trials (see Section 4.1).

sample. This supports the idea that a significant fraction of them still remains unobserved.

In addition to the latter, some relics may escape discovery for other reasons, including: confusion due to bright objects; the presence of a radio halo close to the relic position; low surface brightness as a result of the very extended emission of close-by objects; roundish morphology when located in distant clusters, or simply misclassification, e.g. the relic is too small and is located close to the cluster centre. Despite all these biases, it is evident that the NVSS completeness will mainly depend on relic flux density. It is reasonable to think that objects having flux of about $100 \mathrm{mJy}$ are almost all detected. However, one has to be cautious, yet a few relics of this class do not have been discovered until recently (e.g., PSZ1 G108).

\section{RADIO RELICS IN A COSMOLOGICAL SIMULATION}

\subsection{Simulated galaxy clusters}

In this work, we use the non-radiative MUSIC-2 galaxy cluster sampl ${ }^{3}$ of Sembolini et al. $(2013)$ to generate a set of synthetic radio relic observations which can be compared with the NVSS images. The galaxy cluster sample consists of 282 regions hosting a massive galaxy cluster extracted from the MULTIDARK $N$-body cosmological simulation (Prada et al. 2012). This simulation is performed

${ }^{3}$ music.ft.uam.es

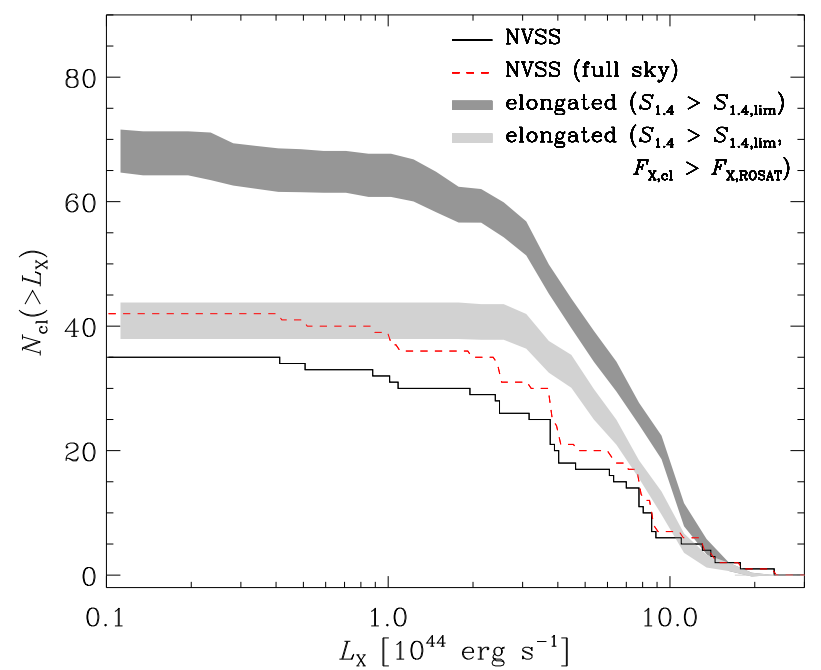

Figure 6. Cumulative number of clusters hosting radio relics versus cluster X-ray luminosity. The original (solid line) and expected (red solid line) NVSS samples are also shown. The latter has been obtained after correcting the original NVSS counts by sky incompleteness as explained in the text. Shaded regions indicate the mean and standard deviation of a set of MUSIC-2 trials (see Section 4.1) for a total radio flux above the effective lower limit of the NVSS.

within the context of the concordance cosmology and comprises a comoving volume of $1 h^{-3} \mathrm{Gpc}^{3}$. Specifically, the adopted cosmological parameters correspond to a flat universe with a matter density of $\Omega_{\mathrm{M}}=0.27$, a baryon density of $\Omega_{\mathrm{b}}=0.0469$, an amplitude of mass fluctuations of $\sigma_{8}=0.82$, a scalar spectral index of $n=0.95$ and $h=0.7$, i.e. the Hubble constant amounts to $70 \mathrm{~km} \mathrm{~s}^{-1} \mathrm{Mpc}^{-1}$.

The zooming technique (Klypin et al. 2001) was used to produce the initial conditions (ICs) necessary for the resimulation of Lagrangian regions within a distance of $6 h^{-1} \mathrm{Mpc}$ around the cluster mass centre at $z=0$. The ICs were evolved until the present time using the parallel TreePM+SPH GadGeT hydrodynamical code (Springel 2005). Additionally, haloes and subhaloes have been identified and characterized using the hybrid MPI+OpenMP parallel halo finder AHF (Knollmann \& Knebe 2009). The mass resolution of these high-resolution regions corresponds to a dark-matter and gas particle mass of $m_{\mathrm{DM}}=9 \times 10^{8} h^{-1} \mathrm{M}_{\odot}$ and $m_{\mathrm{g}}=1.9 \times 10^{8} h^{-1} \mathrm{M}_{\odot}$, respectively. This implies that most of our resimulated massive clusters are typically described with more than a million gas particles. The gravitational softening length in the high-resolution zones was set to $6 h^{-1} \mathrm{kpc}$ both for the gas and dark matter particles.

Several low mass clusters have been found close to the most massive systems in our sample. Therefore, the total number of resimulated clusters considerable exceeds the number of selected cluster volumes. In total, the MUSIC2 sample contains 535 clusters with $M_{\text {vir }}>10^{14} h^{-1} \mathrm{M}_{\odot}$ and more than 2000 group-like objects with virial masses in the range $10^{13}-10^{14} h^{-1} \mathrm{M}_{\odot}$ at $z=0$. Although most of these systems are located outside the virial radius $\left(R_{\text {vir }}\right)$ of our target galaxy clusters any possible interaction between them is consistently described by our simulations. 
The cumulative mass function of the cluster sample between redshifts $0 \leqslant z \leqslant 1$ can be seen in Fig. 2 of Sembolini et al. (2013). The mass completeness limit of the MUSIC-2 dataset goes from $4.5 \times 10^{14} h^{-1} \mathrm{M}_{\odot}$ at $z=1$ to $8.5 \times 10^{14} h^{-1} \mathrm{M}_{\odot}$ at $z=0$ (see their Table 1 ). Since the progenitors of the clusters are also included in the analysis, the final sample is effectively complete at somewhat lower masses. Here, we adopt a low-mass threshold of $5.5 \times 10^{14} h^{-1} \mathrm{M}_{\odot}$.

In what follows, we consider the whole set of 282 resimulated galaxy cluster volumes at 8 different times corresponding to redshifts of $z=0,0.11,0.25,0.33,0.43,0.67,1,1.50$, thus producing a catalogue of 2256 outputs.

\subsection{Cluster X-ray luminosity}

One of the most evident observational features of galaxy clusters is their extended X-ray emission. To compare the simulated clusters with those hosting an NVSS relic it is necessary to assign X-ray luminosities to the simulations. We avoid computing it directly from the simulated galaxy clusters since the non-radiative nature of MUSIC-2 would bias the total luminosity towards artificial higher values. Instead, we use an empirical scaling relation linking the cluster mass to the total X-ray output. We need to compute the rest-frame emission in the $0.1-2.4 \mathrm{keV}$ energy band, as in the case of the observed galaxy clusters listed in Table 1 . To this end, we adopt the scaling relation given by Böhringer et al. (2014)

$$
\frac{L_{500,0.1-2.4}}{10^{44} h_{70}^{-2} \mathrm{erg} \mathrm{s}^{-1}}=\frac{0.1175 E(z)^{\alpha}}{h_{100}^{2-\alpha}}\left(\frac{M_{200}}{10^{14} h_{70}^{-1} \mathrm{M}_{\odot}}\right)^{\alpha},
$$

where $\alpha=1.51, E(z)=\sqrt{\Omega_{\mathrm{M}}(1+z)^{3}+\Omega_{\Lambda}}$ and $M_{200}$ is the cumulative cluster mass within $R_{200}$, i.e. the radius for which the overdensity is 200 times the critical density of the universe. For simplicity, throughout this paper, we will use $L_{\mathrm{X}}$ instead of $L_{500,0.1-2.4}$ to indicate cluster X-ray luminosities within $R_{500}$ in the $0.1-2.4 \mathrm{keV}$ band.

We note that MUSIC-2 clusters are characterized by their virial masses, although masses at different radii are also available. Therefore, to evaluate this scaling relation, we use the $M_{200}$ value corresponding to each cluster. If $M_{200}$ is not available, which only happens a few times per snapshot, we estimate $M_{200}$ from the virial mass. For the cosmological parameters adopted in our simulations, the cumulative density contrast with respect to the critical density of the universe at the radius of virialization is approximately $\Delta=100$ at $z=0$. To convert between $M_{\Delta}$ and $M_{200}$ we assume an NFW profile adopting the halo concentrations of Duffy et al. (2008).

Using this scaling for the effective mass completeness given in the previous section, we estimate that the simulated cluster sample is complete for X-ray luminosities above $2.6 \times 10^{44} \mathrm{erg} \mathrm{s}^{-1}$, adopting an average redshift of $z=0.3$. This result evidently agrees with the comparison of the number of clusters in the NVSS and MUSIC-2 samples. This is shown in Fig. 6, where we plot the cumulative number of clusters hosting radio relics as a function of X-ray luminosity. The curves corresponding to the NVSS and MUSIC-2 samples start to flatten at an X-ray luminosity of about $3 \times 10^{44} \mathrm{erg} \mathrm{s}^{-1}$ in agreement with the X-ray limit estimated above. More details concerning this figure will be presented in Section 4.5 where we will discuss on the relic abundance.

\subsection{Shock finder algorithm}

We identify shock fronts in the simulated galaxy clusters at all available redshifts following Hoeft et al. (2008) and Nuza et al. (2012). In this section, we present a brief description of the shock detection scheme.

For every gas particle we evaluate the pressure gradient and define its shock normal as $\mathbf{n} \equiv-\nabla P /|\nabla P|$. We then search for true shocks by imposing the following conditions: (i) $\nabla \cdot \mathbf{v}<0$, (ii) $\rho_{\mathrm{u}}<\rho_{\mathrm{d}}$ and (iii) $S_{\mathrm{u}}<S_{\mathrm{d}}$, where $\mathbf{v}$ is the velocity field, $\rho$ is the gas density and $S$ its entropy for the upstream and downstream regions respectively. To determine the Mach number we use the Rankine-Hugoniot equations for hydrodynamical shocks (see e.g., Landau \& Lifshitz 1959) for each one of the above conditions and then take the minimum resulting value as a conservative estimate. This is done in order to avoid an overestimation of the Mach number that could lead to strong spurious radio emission.

\subsection{Magnetic field model}

Very little is known about the strength and structure of magnetic fields in radio relics. One of the most stringent lower limits for the field strength has been derived by Nakazawa et al. (2009) for the relic in A3667. Based on upper limits of the IC emission from the relic region they concluded that the field strength must exceed $1.6 \mu \mathrm{G}$. In radio relics, magnetic fields could be dominated by those generally present in the ICM and then compressed by the shock front. In addition, the field might be amplified, for instance, by upstream instabilities (Guo et al. 2014a) or downstream turbulence (Donnert et al. 2016).

It is beyond the scope of this work to model relic magnetic fields in detail. Therefore, we adopt a simple parametrization linking the magnetic field strength $B$ to the local electron density $n_{\mathrm{e}}$. Following Nuza et al. (2012), we assume a scaling of the form

$$
B=B_{0}\left(\frac{n_{\mathrm{e}}}{10^{-4} \mathrm{~cm}^{-3}}\right)^{\eta},
$$

in agreement with previous works (see e.g., Dolag et al.|2001, Stasyszyn et al. 2010). The parameters adopted correspond to the best-fit model of Bonafede et al. (2010) for the Coma cluster, i.e. $B_{0}=0.8 \mu \mathrm{G}$ and $\eta=1 / 2$. These values lead to magnetic field strengths of the order of $1 \mu \mathrm{G}$ in cluster outskirts. It is worth noting that the field strength adopted here is generally below the lower limits derived by Nakazawa et al. (2009) for A3667 and van Weeren et al. (2010) for the 'Sausage' relic, namely 1.6 and $5 \mu \mathrm{G}$ respectively. However, the field strength parameter $B_{0}$ and the electron acceleration efficiency in our model (see below) are basically degenerated in radio luminosity. This implies that, if magnetic field strengths in relics are higher than adopted here, the true acceleration efficiency will be lower.

\subsection{Lighting up the shocks}

As for the magnetic field, our knowledge about the origin and acceleration mechanism of the relativistic electrons 
which give rise to the observable synchrotron emission is incomplete. It is generally assumed that diffusive shock acceleration (DSA) at the merger shock fronts leads to the relativistic energies of the 'observable' electrons, although many details of the acceleration mechanism are under discussion. For instance, pre-existing populations of cosmic ray electrons may significantly enhance the acceleration efficiency (Kang et al. 2012). Alternatively, shock drift acceleration may result in an efficient mechanism to accelerate electrons at low Mach number shocks (Guo et al. 2014a).

Since up to now the details of the acceleration mechanism are unknown, we follow the general framework of Hoeft \& Brüggen (2007). This model is based on two main assumptions: (i) the slope of the energy distribution of the accelerated electrons follows the predictions of test particle DSA and (ii) only a fixed fraction, $\xi_{\mathrm{e}}$, of the energy dissipated at the shock front is used to accelerate the electrons. The resulting spectrum of relativistic electrons may be considered as the average outcome of an acceleration process which is, in detail, much more complicated than test particle DSA. Hoeft \& Brüggen (2007) compute the total radio emission coming from a shock assuming that electrons accelerated at the front are advected with the downstream plasma. In this region, electrons loose energy via synchrotron and IC emission, the latter being caused by collisions with cosmic microwave background (CMB) photons.

As a result, the total radio luminosity is given by a few parameters, namely, the Mach number of the shock and the thermodynamical properties and magnetic field strength of the downstream plasma. For the smoothed-particle hydrodynamics (SPH) particles we identify those which are located at a shock front using the shock finder described above. Since the cooling of electrons takes place on much smaller length scales than is possible to resolve, we can attribute the entire radio luminosity of simulated relics to $\mathrm{SPH}$ particles at the shock front. Therefore, the output radio luminosity per unit frequency contributed by every shocked particle will also be related to its associated shock area element. The radio power per unit frequency contributed by an SPH gas particle $i$ can be written as

$$
\begin{aligned}
P_{\nu, i}=6 & \times 10^{34} \mathrm{erg} \mathrm{s}^{-1} \mathrm{~Hz}^{-1} \frac{A_{i}}{\mathrm{Mpc}^{2}} \frac{n_{\mathrm{e}, i}}{10^{-4} \mathrm{~cm}^{-3}} \\
& \times \frac{\xi_{\mathrm{e}}}{0.05}\left(\frac{\nu}{1.4 \mathrm{GHz}}\right)^{-\frac{s_{i}}{2}}\left(\frac{T_{\mathrm{d}, i}}{7 \mathrm{keV}}\right)^{\frac{3}{2}} \\
& \times \frac{\left(B_{\mathrm{d}, i} / \mu \mathrm{G}\right)^{1+\frac{s_{i}}{2}}}{\left(B_{\mathrm{CMB}} / \mu \mathrm{G}\right)^{2}+\left(B_{\mathrm{d}, i} / \mu \mathrm{G}\right)^{2}} \Psi\left(\mathcal{M}_{i}\right),
\end{aligned}
$$

where $A_{i}$ represents the surface area associated to the particle, $n_{\mathrm{e}, i}$ is the electron density, $\xi_{\mathrm{e}}$ is the electron acceleration efficiency, $s_{i}$ is the slope of electron energy distribution as given by DSA, $T_{\mathrm{d}, i}$ is the post-shock temperature, $B_{\mathrm{d}, i}$ is the post-shock magnetic field, $B_{\mathrm{CMB}}$ is the magnetic measure of the CMB energy density and $\Psi\left(\mathcal{M}_{i}\right)$ is a function that depends on the shock strength. The area corresponding to each SPH particle is proportional to the square of the smoothing length divided by the number of particles within the kernel. The electron acceleration efficiency denotes the fraction of energy dissipated at the shock front that is transferred to suprathermal particles. We note that, in practice, this parameter acts as a normalization factor that does not depend on the strength of the shock. The latter is taken into account by the $\Psi\left(\mathcal{M}_{i}\right)$ function which, for weak shocks in the range $\mathcal{M}_{i} \gtrsim 2-4$, gives an additional factor of about 0.01-0.5 (see Fig. 4 of Hoeft \& Brüggen 2007). Shock fronts with lower Mach numbers have, in general, very low radio luminosities due to the steep decline of $\Psi\left(\mathcal{M}_{i}\right)$. Within this context, an effective Mach-dependent acceleration efficiency can be constructed as the product of these two quantities, i.e., $\xi_{\mathrm{e}}^{\prime}(\mathcal{M}) \equiv \xi_{\mathrm{e}} \Psi(\mathcal{M})$. The interested reader is referred to Hoeft \& Brüggen (2007) and Hoeft et al. (2008) for more details concerning our radio emission scenario.

The combination of shock finder, magnetic field prescription and radio luminosity model allows us to 'illuminate' merger shocks in the simulation. Although this model clearly simplifies the complex processes taking place in relics, it allows us to estimate the position and morphology of merger shocks in our cosmological simulation in a way which suits our needs. Throughout this paper, we assume $\xi_{\mathrm{e}}=5 \times 10^{-5}$ as a working efficiency value which is enough to reproduce the number of NVSS clusters with $S_{1.4, \text { tot }}=100 \mathrm{mJy}$ and is also consistent with the findings of Nuza et al. (2012). A more detailed discussion concerning relic abundance will be presented in Section 4.5

\subsection{Mock cluster and radio relic observations}

Our aim is to produce a set of mock relics that can be used to study the properties predicted by our model, as well as to compare results with available observations. Here, the mock instrumental parameters will be chosen to resemble those of an NVSS-like survey, as described in Section 2.2. Therefore, in what follows, we will consider an observational frequency of $\nu_{\text {obs }}=1.4 \mathrm{GHz}$, a telescope beam size of $45^{\prime \prime}$ and a survey sensitivity of $\sigma_{\mathrm{NVSS}}=0.45 \mathrm{mJy}_{\mathrm{beam}^{-1}}$.

We generate a set of synthetic observations for the galaxy clusters in our sample from surface brightness maps obtained by projecting all cluster radio emission inside $2 \times R_{\text {vir }}$ onto a plane. In all cases, the rest frame radio frequency has been $k$-corrected to obtain our mock observations at frequency $\nu_{\mathrm{obs}}$. To produce radio images resembling those obtained by actual radio telescopes all projected emission has been convolved with a Gaussian filter at a scale $\theta_{\text {FWHM }}$ which corresponds to the beam size resolution at the given redshift of the cluster. Then, the emission has been converted into flux per beam. We identify island boundaries using $2 \times \sigma_{\text {NVSS }}$ contour lines and discard all islands with a flux below $8 \times \sigma_{\mathrm{NVSS}}=3.6 \mathrm{mJy}$, as done for the NVSS sample.

In our images, the X-ray emission of the clusters is shown as colour-coded maps. The X-ray luminosity of each particle has been computed using the software XSPEC adopting a MEKAL emission model. We note that X-ray maps are only used to illustrate the dynamical state of the cluster and no attempt is made of computing the emission directly from the simulations. This is due to the fact that non-radiative simulations tend to overestimate the bremstrahlung luminosity output since they lack the necessary energy feedback to lower the ICM gas density (Nagai et al. 2007).

Fig. 7 shows some examples of relics found in the MUSIC-2 cluster sample which are 'observed' assuming NVSS specifications. All clusters show clear evidence of recent merger activity in the X-ray surface brightness distribution. Some of these clusters host single, double or more 

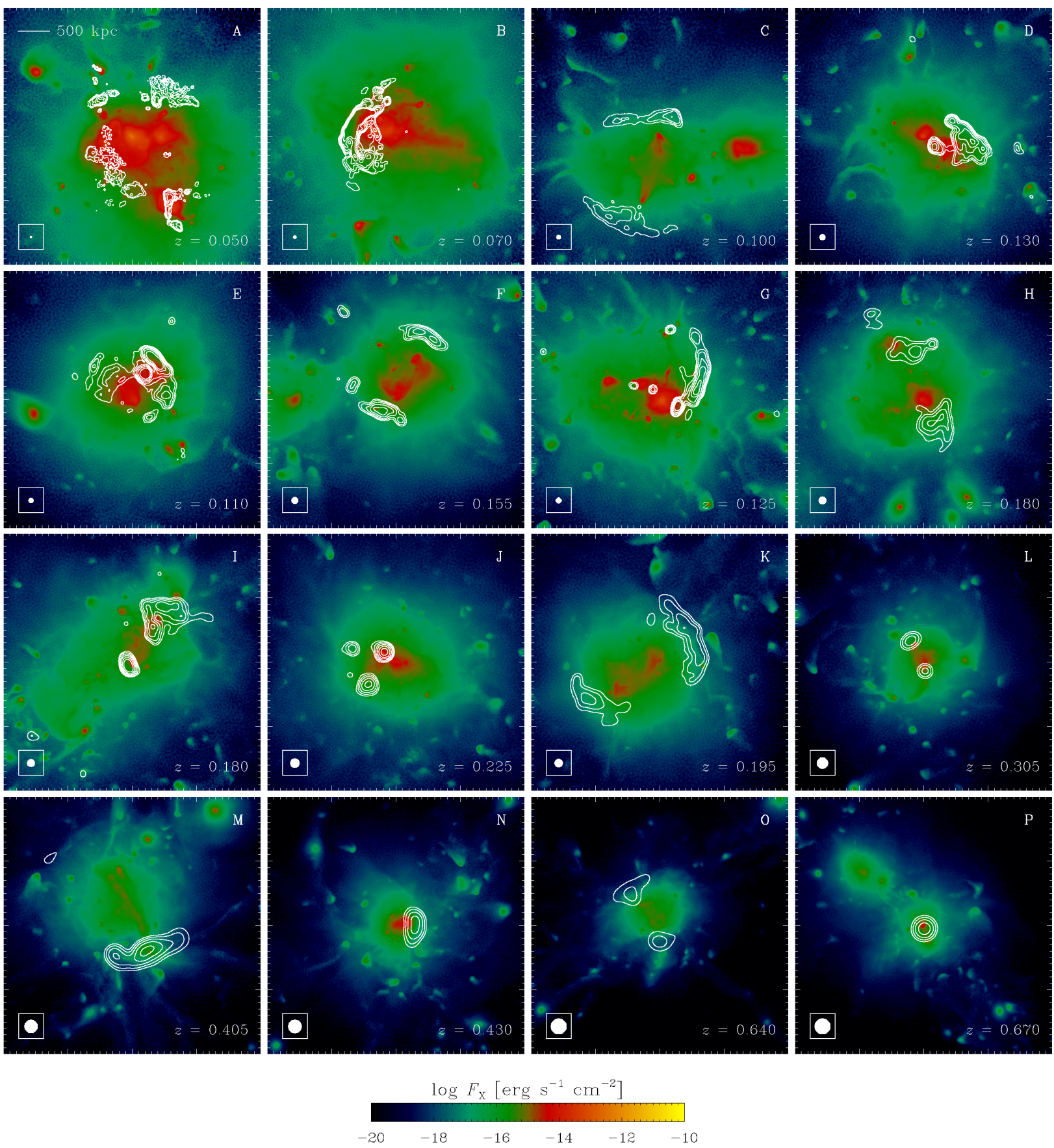

Figure 7. Examples of mock radio relic observations for clusters at different redshifts. The colour scale indicates the X-ray surface brightness of simulated clusters. Synthetic relic surface brightness at $1.4 \mathrm{GHz}$ observing frequency is shown using contours drawn at $[2,4,8,16, \ldots] \times \sigma_{\text {NVSS }}$ with $\sigma_{\text {NVSS }}=0.45 \mathrm{mJy}$ beam $^{-1}$. Every panel spans a proper area of $4 \times 4 \mathrm{Mpc}^{2}$ whereas the filled white circles indicate the FWHM beam area. A large morphological diversity of relics can be observed.

irregular relic structures. For instance, clusters 'C', 'F', and ' $\mathrm{K}$ ', show nice double gischt morphologies as those found in observations. Very often the two cores of the progenitor clusters are well visible in the X-ray maps. An spectacular example of a single relic is shown in cluster ' $G$ '. Large extended objects are also seen at high redshift, an example of which is cluster ' $\mathrm{M}$ ' that hosts a quasi-linear radio structure of about $1 \mathrm{Mpc}$ long at $z=0.405$. Other clusters show that radio relic morphologies may significantly deviate from classical textbook examples, i.e. elongated structures tangentially oriented in the cluster outskirts. For instance, the relic in cluster ' $\mathrm{B}$ ' is located very close to the cluster centre with a morphology resembling the enigmatic object found in Abell 523. Another example is the complex relic in cluster ' $\mathrm{D}$ ', which is not at all positioned along the apparent merger axis. Complex merger scenarios can give rise to peculiar emission features such as those seen in cluster 'A'. Moreover, a line-of-sight along the merger axis, or close, can 

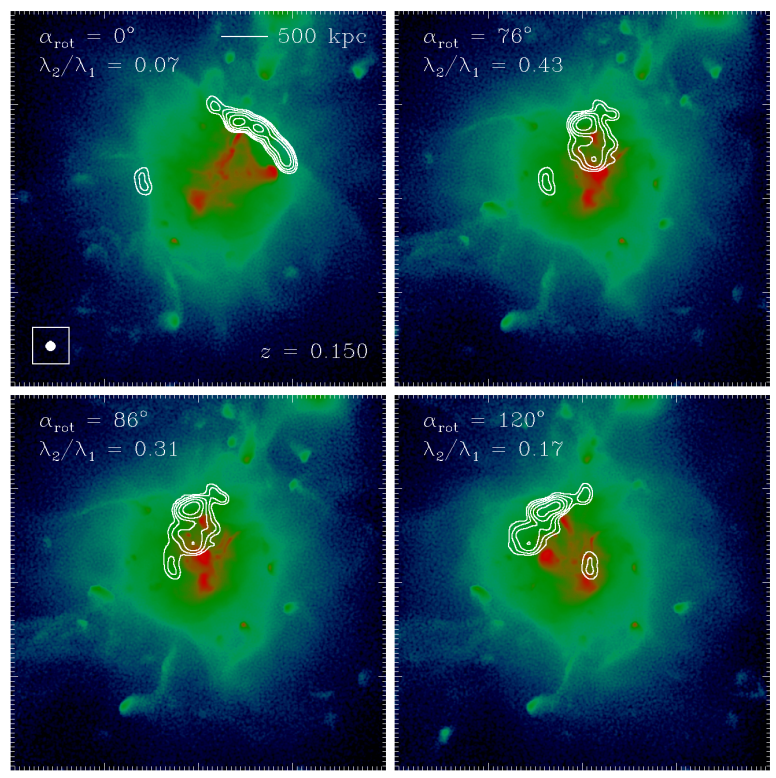

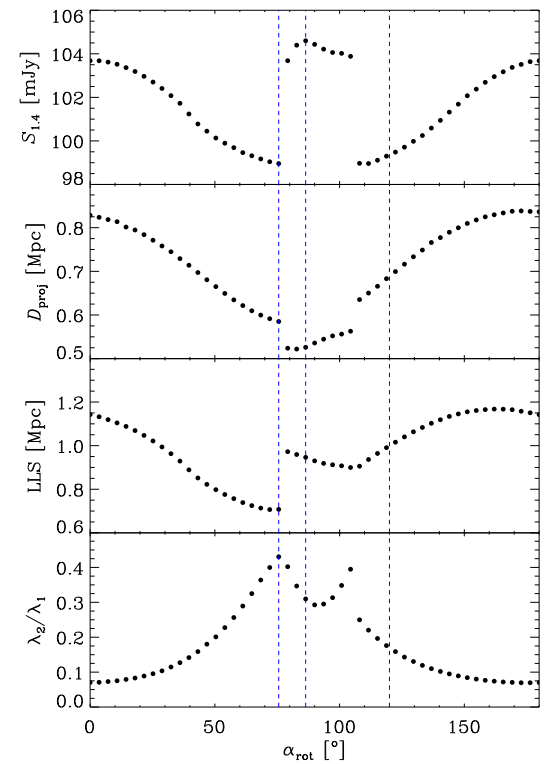

Figure 8. Left-hand panel: Idem as Fig. 7 for mock observations of one of the simulated clusters at $z=0.15$ and four different rotation angles, $\alpha_{\text {rot }}$. Also shown is the shape parameter of the largest relic in each panel. Right-hand panel: From top to bottom: flux density, projected distance, largest linear size, and shape parameter of the largest relic island as a function of rotation angle. The vertical dashed lines indicate the rotation angles shown in the left-hand panel.

reveal how relic surfaces can break into several pieces, as shown, e.g., in cluster 'E'. Finally, small and/or poorly resolved emission features typically appear as more roundish objects as in clusters ' $\mathrm{J}$ ', 'L' and ' $\mathrm{P}$ '.

We speculate that a significant fraction of the roundishlike islands are caused by spurious effects in the simulation, with possibly several origins. The simulation has been carried out without any heating of the ICM except for the dissipation of structure formation shocks. It is known that this kind of treatment underestimates the temperature of the ICM, most significantly in the cluster centre. Therefore, owing to the lower sound speed, fast moving dense clumps may more easily generate a small shock. Moreover, since SPH is intrinsically noisy and the Mach number depends in a very non-linear way on the particle properties, one may also find some outliers. Fig. 8 shows how the morphology and other island parameters change with viewing angle. To study this, we have rotated one of the clusters in our MUSIC-2 set along the vertical axis. We plot four panels, one for each rotation angle. This particular example consists of a large linear gischt structure of about $1.2 \mathrm{Mpc}$ long located at about 0.9 $\mathrm{Mpc}$ from the cluster centre when the relic is seen edgeon, i.e. $\alpha_{\text {rot }}=0^{\circ}$. A hint of a second relic structure in the opposite side of the cluster can also be seen. This scenario changes dramatically when rotating the cluster. The righthand panel of Fig. 8 plots the evolution of several island parameters for the main relic as a function of rotation angle. As expected, radio flux, projected distance and LLS decrease as the rotation angle tends to $90^{\circ}$. The opposite is true for the shape parameter, meaning that, when the relic is seen face-on, its shape appears rounder to the observer. Moreover, when the projected contours of the two radio features merge (separate) at $\alpha_{\text {rot }} \approx 80^{\circ}\left(105^{\circ}\right)$ there are clear discontinuities in the island parameters. These results show that special care must be taken when interpreting observations as projection effects can significantly affect the location and morphological properties of relics.

We find that the MUSIC-2 cluster sample leads to a large variety of radio relics, covering textbook examples of single and double relics to systems with a very peculiar morphology. In the next section, we will use these relics to statistically compare their location, morphology and emission properties with those found in NVSS.

\section{PROPERTIES OF THE SAMPLES}

Many spectacular radio relics have been observed and their properties studied in considerable detail. However, we can still only speculate if all relics are caused by merger shocks in a homogeneous way. Here, we would like to know if the radio luminosities of relics found in NVSS, their relation to the X-ray cluster luminosity, their size, morphology and location within galaxy clusters can be reproduced by the relics identified in the MUSIC-2 cluster sample. The location, size and morphology of the simulated relics are determined by the shock fronts. Hence, both the merger history of galaxy clusters and the evolution of the ICM determine their properties within this scenario.

\subsection{Constructing a representative cluster sample}

First of all, we need to remember that the sample of relics identified in NVSS is flux limited, besides other limitations discussed in Section 2.4 Since the number of known relics is still small we cannot easily derive a volume limited sample comprising a sufficient large number of relics by considering only a small redshift range. Therefore, in our modelling of 


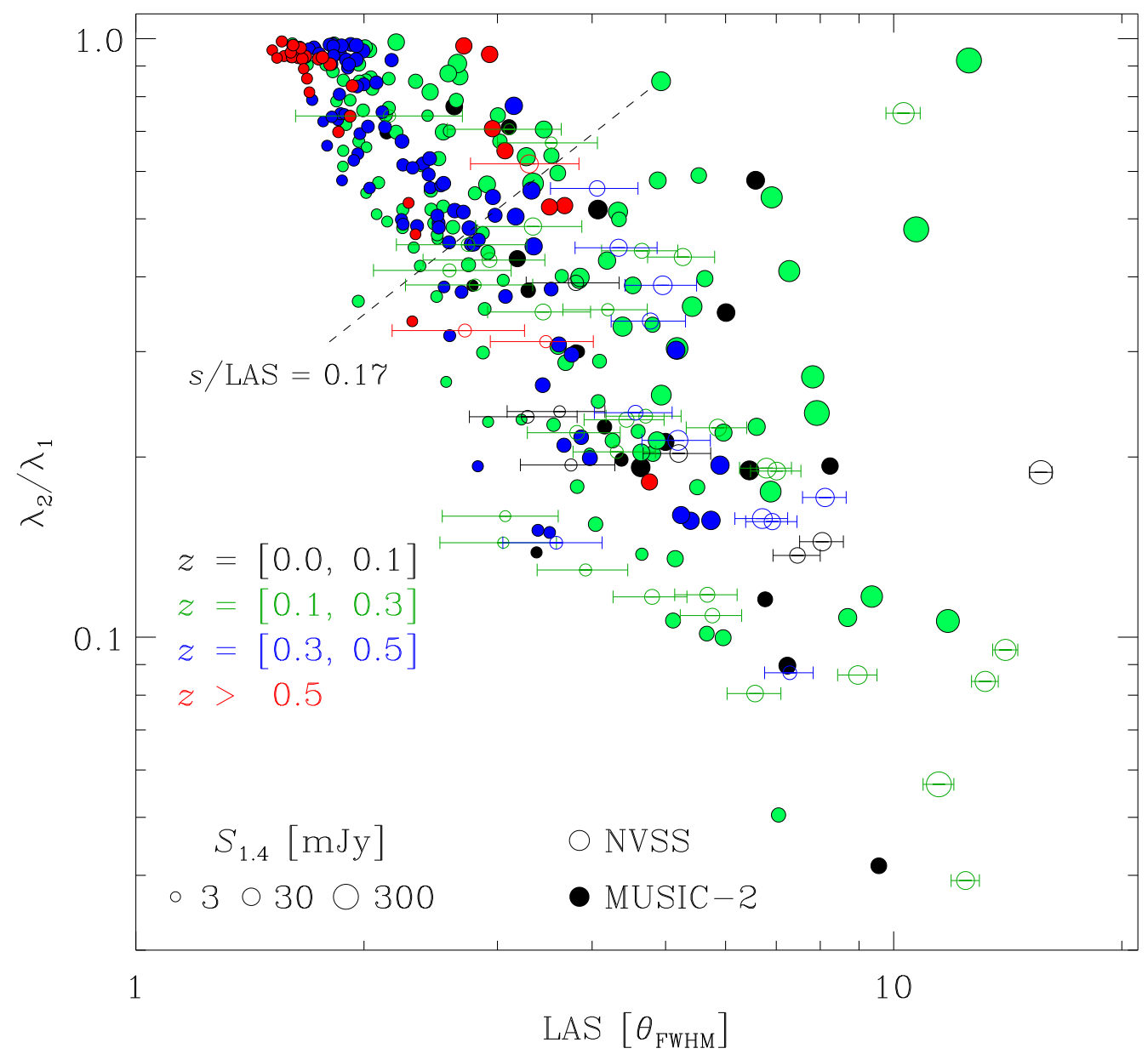

Figure 9. Shape parameter, $s \equiv \lambda_{2} / \lambda_{1}$, versus the largest angular size (LAS) for the NVSS (empty circles) and MUSIC-2 (solid circles) relic samples. Symbol sizes are scaled according to radio flux. Redshift bins are indicated using different colours. We distinguish the 'elongated' from the 'small-roundish' islands using the empirical criterion $s /$ LAS $<0.17$ (see dashed line).

relic observations we reproduce the NVSS flux limited sample.

Radio relics in the redshift interval $z \in\left[z_{i}, z_{i+1}\right]$ are located within a comoving volume of

$$
V_{\mathrm{c}}\left(z_{i}, z_{i+1}\right)=\frac{4 \pi}{3}\left(R_{\mathrm{c}}^{3}\left(z_{i+1}\right)-R_{\mathrm{c}}^{3}\left(z_{i}\right)\right),
$$

where $R_{\mathrm{c}}$ denotes the comoving radial distance and a flat universe is assumed. In order to generate the mock relic sample we proceed as follows. We define a sequence of shells around the observer with comoving volumes according to Eq. 10 For each redshift interval, we take the snapshot of the simulation with the closest redshift to the shell mean value. The ratio of the shell volume to the simulation one determines how many clusters from the MUSIC-2 sample should be chosen to 'populate' the shell. Then, we randomly pick these clusters from the sample.

Since the redshift distribution within the shell is not uniform, the redshift of the selected cluster is randomly chosen from the $\left[z_{i}, z_{i+1}\right]$ interval assuming a probability proportional to the comoving volume. Therefore, the normalized probability density of finding a cluster at redshift $z$ within the shell can be written as

$$
\mathcal{P}(z)=\frac{1}{V_{\mathrm{c}}\left(z_{i}, z_{i+1}\right)} \frac{\mathrm{d} V_{\mathrm{c}}(0, z)}{\mathrm{d} z}
$$

The cluster selection procedure implies that only a few clusters are finally drawn from simulation snapshots at low redshifts. At higher redshifts the situation is different. For example, the simulation snapshot at $z=0.43$ serves as a proxy for the redshift interval $z \in[0.38,0.55]$. In this case, the comoving volume of the shell amounts to $23.4 \mathrm{Gpc}^{3}$, indicating that every cluster in this snapshot needs to enter many times in the sample. To avoid duplicating the same relic parameters, we randomly rotate the clusters in every iteration (see Fig. 8 for the effect of rotation on the relic properties such as morphology and flux). The fact that clusters at $z \gtrsim 0.5$ enter the sample a few tens of times shows the need of a large simulation volume for our purpose. In this respect, the cosmological box and simulation technique of the MUSIC-2 set turn out to be a good compromise: at the mean redshift of our radio relic samples, i.e. $z \sim 0.2-0.3$, clusters enter in the mock catalogues only a few times at 


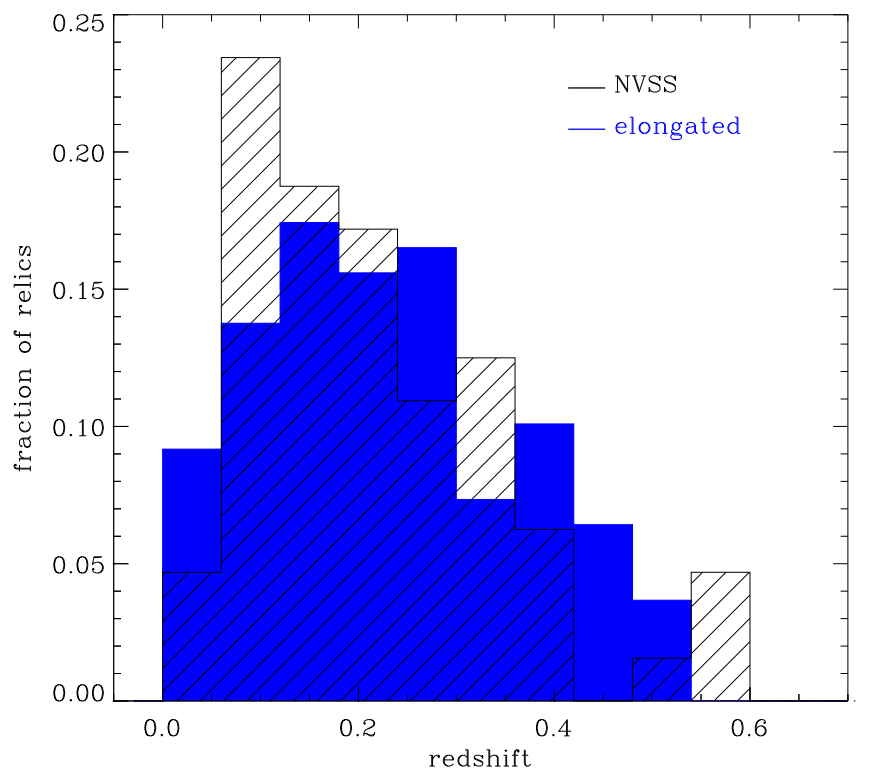

Figure 10. Redshift distribution of the NVSS and 'elongated' relic samples (dashed and blue histograms, respectively).

maximum, while the zoomed method permits to resolve the shock fronts in a reasonable way.

As a result of this approach, the resulting mock relic sample will represent a unique realisation of the selection process and, therefore, relic properties will depend on which clusters are finally included in the sample. Then, if required, we can average over several realisations, e.g. to get mean number counts that can be compared to observations. In what follows, we start by contrasting the properties of islands before considering the abundance and flux distribution of the relic sample.

\subsection{The radio islands}

In Section 2.2 we have shown that relics, more precisely the radio emission islands, found in the NVSS images show a strong correlation between shape and the largest angular size (LAS). As a first step of our comparison, we investigate if the NVSS island sample shows a similar correlation. As a reminder, we summarize the relic selection criteria considered in our NVSS sample: (i) all islands with a flux below $8 \times \sigma_{\text {NVSS }}=3.6 \mathrm{mJy}$ are discarded since image noise may have a significant impact on their morphology, and (ii) clusters at $z<0.05$ are also discarded since, at these redshifts, the recovery of relics with a typical extent of $1 \mathrm{Mpc}$ already starts to be affected by the inner uv-gap of the VLA D configuration.

We can now discuss the results of Fig. 9. This plot shows the distribution of shape parameter versus LAS for both samples. Evidently, the mock sample displays a similar (anti)correlation as observations. In contrast with the NVSS relics, the simulated sample shows many objects above the empirical threshold $s / \mathrm{LAS}=0.17$. In the following analysis, we subdivide the simulated relics into 'small-roundish' and 'elongated' samples which comprise the islands above and below the threshold, respectively. When comparing to NVSS

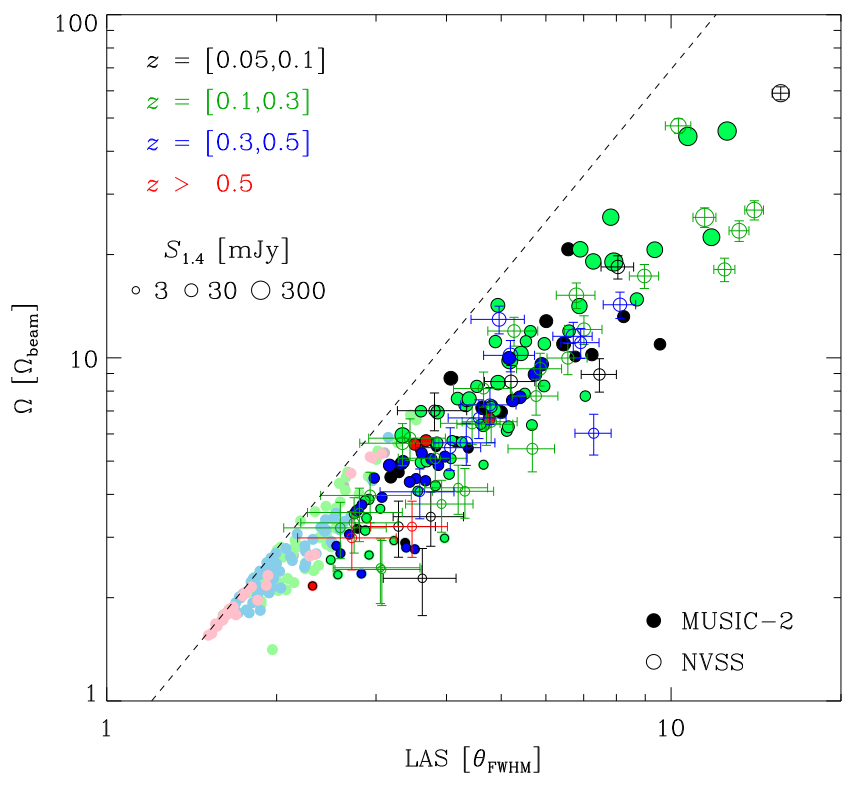

Figure 11. Solid angle, $\Omega$, versus the largest angular size, LAS, for the NVSS and mock samples. Shown are the NVSS islands (empty circles) and the 'elongated' (solid circles) and 'smallroundish' (light solid circles) mock relic samples. Symbol sizes for the NVSS and 'elongated' samples are scaled according to radio flux. In all cases, redshift bins are indicated using different colours. For reference, the relation for a circular object is shown as a dashed line.

data we will consider only the 'elongated' sample since the small-roundish objects are mostly found in the mock observations (see the discussion in Section 5.

It is interesting to note, however, that the mock relic sample shows only very few large roundish objects which may originate from relics seen face-on. It has been speculated that some giant radio haloes, which are centred on the X-ray cluster emission and follow its morphology, might result from radio relics seen perfectly face-on. Our simulation confirms that such a situation is very rare and cannot significantly contaminate the giant halo sample.

The redshift distribution of NVSS and 'elongated' relics is shown in Fig. 10 To compare with NVSS data in an unbiased way, we need to exclude the 'small-roundish' islands. Both distributions are quite similar with mean redshift values of 0.23 and 0.21 for the simulated and NVSS samples, respectively. The main difference between the two is that we find a smaller relic fraction in the mock sample at low redshift. This can be explained by the fact that many lowredshift relics in NVSS reside in low-mass clusters, which are not included in MUSIC-2. However, at $z \sim 0.4-0.5$ we find a higher relic fraction in comparison with observations. We speculate that this is caused by the fact that some highredshift relics in NVSS may escape discovery since their host galaxy clusters are too faint in X-ray to be identified: most of the observed clusters are above the ROSAT X-ray flux detection limit. In Sections 4.3 and 4.5 , we will give more details concerning this point.

Fig. 11 shows the correlation between solid angle and LAS for the mock (solid circles) and NVSS (open circles) samples. Unless otherwise stated, besides the 'elongated' 


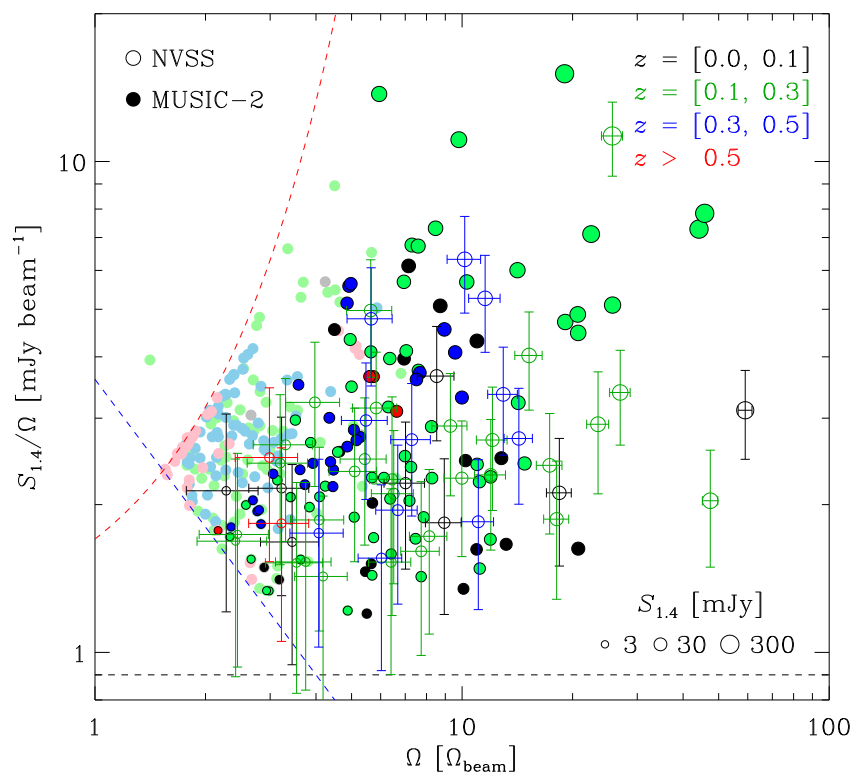

Figure 12. Average surface brightness versus solid angle, $\Omega$, for the relic samples. Shown are the NVSS islands (empty circles) and the 'elongated' (solid circles) and 'small-roundish' (light solid circles) mock relic samples. Symbol sizes for the NVSS and 'elongated' samples are scaled according to radio flux. In all cases, redshift bins are indicated using different colours. For reference, limits associated to the observation process are also drawn, namely, the maximum flux given by Eq. 2 (red dashed line); the cut associated to the minimum flux density condition of an island, i.e. $S_{1.4, \text { min }}=8 \times \sigma_{\mathrm{NVSS}}$, and the minimum possible surface brightness of a beam, i.e $2 \times \sigma_{\text {NVSS }}$, where $\sigma_{\text {NVSS }}$ is the NVSS sensivity.

simulated sample, in what follows we will also show the 'small-roundish' islands using lighter colours. For comparison, the relation for a circle, $\Omega_{\text {circ }}$, is shown as a dashed line. As seen in the figure, the NVSS and 'elongated' samples show a similar distribution. In particular, the mean solid angles of the distributions are essentially the same but the LAS are slightly higher in the NVSS case. For the NVSS sample the filling factor, $\Omega / \Omega_{\text {circ }}$, amounts to about $35 \%$.

Fig. 12 shows the average surface brightness versus the solid angle of the islands. The 'elongated' and NVSS scatter plots nicely match, being their median surface brightnesses 2.46 and $2.40 \mathrm{mJy}^{\text {beam }}{ }^{-1}$, respectively. The lowest possible surface brightness is set by the adopted island boundary, i.e. $2 \times \sigma_{\text {NVSS }}$ (black dashed line). The Gaussian smoothing in both the NVSS images and the mock observations imposes a maximum surface brightness as a function of solid angle given by Eq. 2 (red dashed line). Finally, an additional limit comes from our restriction to consider only islands above $8 \times$ $\sigma_{\text {NVSS }}$ (blue dashed line). These conditions evidently limit the observed surface brightness. Still, it is remarkable that both the NVSS and mock island samples reside in a narrow range of average brightness. This fact may reflect that relics are not compact: even if they comprise bright areas, there are also regions with low surface brightness (see e.g., the 'Toothbrush' relic in Fig. 11. As a result, the deeper the observations are, the more extended the relics may become.

Many authors have reported a correlation between radio power and LLS of relics (e.g., Feretti et al. 2012 Bonafede et al. 2012). Fig. 13 shows this correlation for the NVSS and mock relic samples. The 'elongated' and NVSS samples agree reasonably well. As seen before for the LAS, the 'elongated' mock sample has slightly smaller sizes than observations, with a mean LLS value of $0.7 \mathrm{Mpc}$ in comparison to $0.82 \mathrm{Mpc}$ for the NVSS. If one considers the whole mock relic sample, many objects have radio powers above the median luminosity of the NVSS as a result of the more roundish shape of some of the islands (see Fig. 11). To understand the origin of this correlation, we estimate a lower flux density limit for each redshift bin. To convert LLS into LAS, we adopt the mean redshift of each bin. By using the solid angle versus LAS correlation, we are able to obtain the average area related to LLS. Since the flux density has to be larger than $2 \times \sigma_{\mathrm{NVSS}}$, we obtain a minimum flux limit which we then convert into rest-frame radio luminosity (dashed-lines colour-coded by redshift). We further indicate a diameter of $1.5 \times \theta_{\text {FWHM }}$ adopting the corresponding mean redshift value in each case (vertical solid lines). Basically, for each redshift bin, all islands are above the derived lower-limit radio power estimates. This indicates that the evident correlation between relic luminosity and LLS actually originates from the fact that all observed relics display a similar average surface brightness.

\subsection{Relics in distant clusters}

For each cluster, we determine its radio flux as the sum of all island fluxes. Hence, we can investigate the X-ray flux versus total radio flux correlation, which is shown in Fig. 14. The $\mathrm{X}$-ray flux is estimated from the luminosity via

$$
F_{\mathrm{X}}=\frac{L_{\mathrm{X}}(1+z)}{4 \pi d_{\mathrm{L}}^{2}}
$$

where $d_{\mathrm{L}}$ denotes the luminosity distance. For simplicity, we do not consider here any $k$-correction.

It is very well visible in the plot, that some clusters are 'aligned'. This is caused by the fact that we considered the same simulation snapshot of a cluster multiple times, using an arbitrary cluster rotation and a slightly different redshift in every iteration. We note that this effect mainly occurs for clusters in the 'small-roundish' sample as they enter more times in the relic list as a result of their higher mean redshift. Interestingly, this shows that the $1 h^{-1} \mathrm{Gpc}$ simulation box we are considering here is only marginally sufficient to simulate radio relics in a representative cosmological volume for the sensitivity limits applied.

Evidently, most of the clusters in the NVSS sample are above or in the vicinity of the ROSAT detection limit. In contrast, many of the clusters of the mock sample are significantly below, which correspond to systems at redshift $z \gtrsim 0.3$. We note that the MUSIC-2 cluster sample comprises rather massive clusters, hence, a low X-ray flux can only originate from distant clusters. The fact that the 'smallroundish' sample has no NVSS counterpart and mainly populates distant clusters with low X-ray flux suggests that those relics are notoriously difficult to discover: they are small, faint and primarily reside in clusters not yet found. As noted earlier, some of these objects might be spurious. We will discuss this further below.

The correlation between radio power and cluster X-ray luminosity is well known. We show the resulting scatter plot 


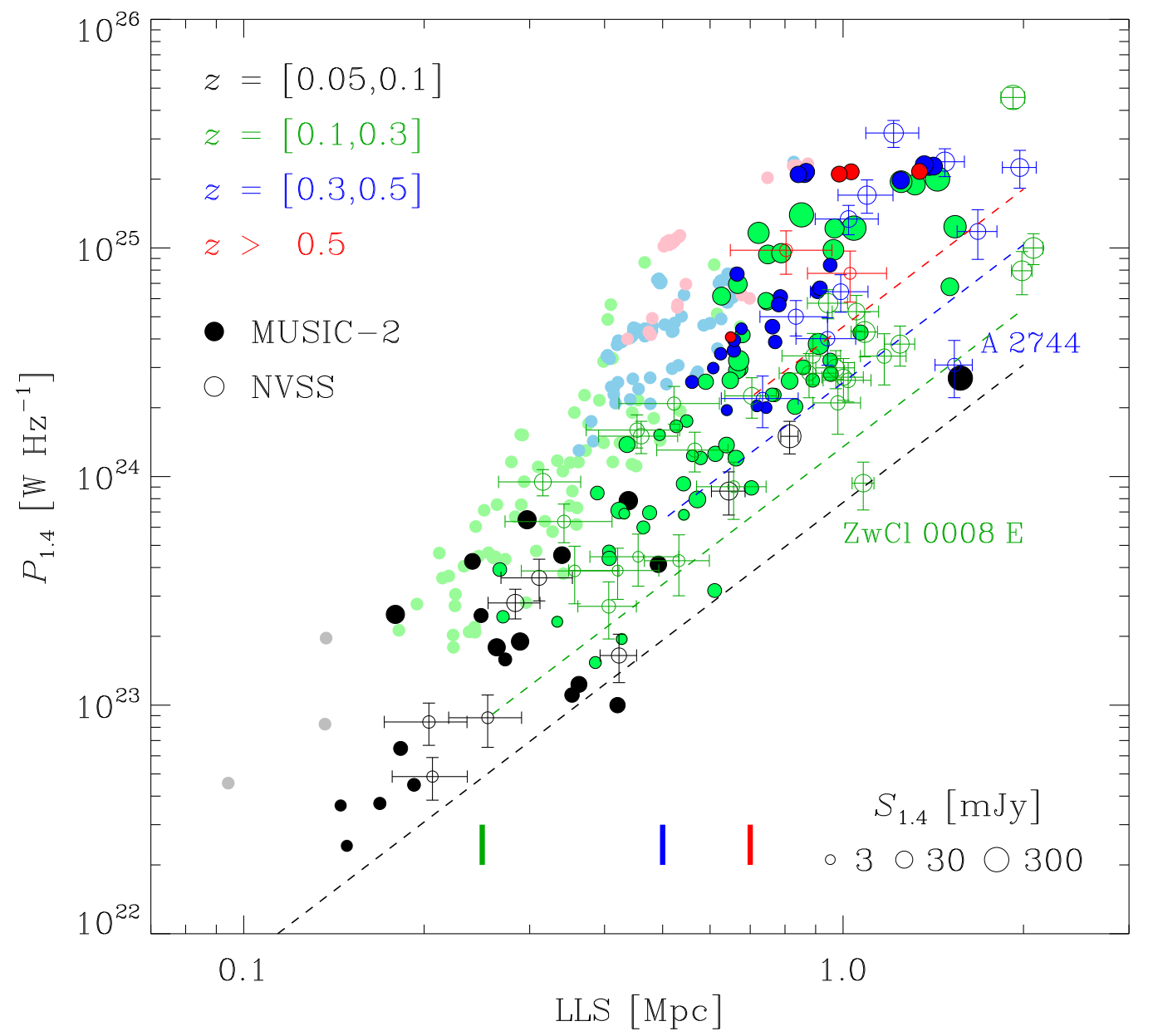

Figure 13. Radio power, $P_{1.4}$, versus largest linear size, LLS, for the relic samples. Shown are the NVSS islands (empty circles) and the 'elongated' (solid circles) and 'small-roundish' (light solid circles) mock relic samples. Symbol sizes for the NVSS and 'elongated' samples are scaled according to radio flux. In all cases, redshift bins are indicated using different colours. For reference, redshift-dependent lower limits for the flux density in the $P_{1.4}$ - LLS plane are shown as colour-coded dashed lines (see text). Additionally, we also indicate the corresponding spatial scales for $1.5 \times \theta_{\mathrm{FWHM}}$ at the same redshifts, where $\theta_{\mathrm{FWHM}}$ is the NVSS beam angular size (vertical solid lines).

for the NVSS and the mock relic samples in Fig. 15. For comparison, we also include the best-fit relation of de Gasperin et al. (2014) after scaling cluster masses to the virial radius (see Section 3.2 and transform to X-ray luminosities using the mass-luminosity relation of Eq. 7. Also shown are the limits arising from the flux density threshold of $8 \times \sigma_{\mathrm{NVSS}}$ and the ROSAT X-ray flux limit by adopting the mean redshift of each bin. It is worth noting that the ROSAT limit is not strict as several clusters below this threshold are known to host relics. In any case, the latter suggests that this correlation is to a large extent determined by the detection thresholds.

\subsection{Relic orientation and projected distance to cluster centre}

In Fig. 16 we study the relation between relic shape and orientation. The latter is characterised by the angle $\phi$ between the position and elongation axis of relics as explained in Section 2.2.2 (see also Fig. 1). The 'elongated' MUSIC-2 and observed relic samples display a very close agreement. In general, symbols tend to cluster at larger orientation angles, more significantly at $\phi \gtrsim 70^{\circ}$; evidently, this effect is stronger at the lowest shape parameters. As shape values increase (the 'small-roundish' MUSIC-2 sample is shown using gray filled circles), the clustering of data smooths out, eventually turning into a uniform distribution that spans all possible orientations. This is most evident at shape values of $\lambda_{2} / \lambda_{1} \gtrsim 0.6$, as the islands become roundish and devoid of any preferred orientation.

The angle and shape distributions for the 'elongated' and observed relic samples (upper and right-hand histograms in Fig. 16 are also shown. In both cases, a similarity between the simulated and observed distributions can be seen. In particular, for the orientation angle distribution, the simulated and observed histograms clearly peak towards angles close to $90^{\circ}$ confirming previous findings (van Weeren et al. 2011). The 'elongated' sample is in remarkable agreement with NVSS observations supporting the idea that relic orientation is indeed linked to the direction of the merging 


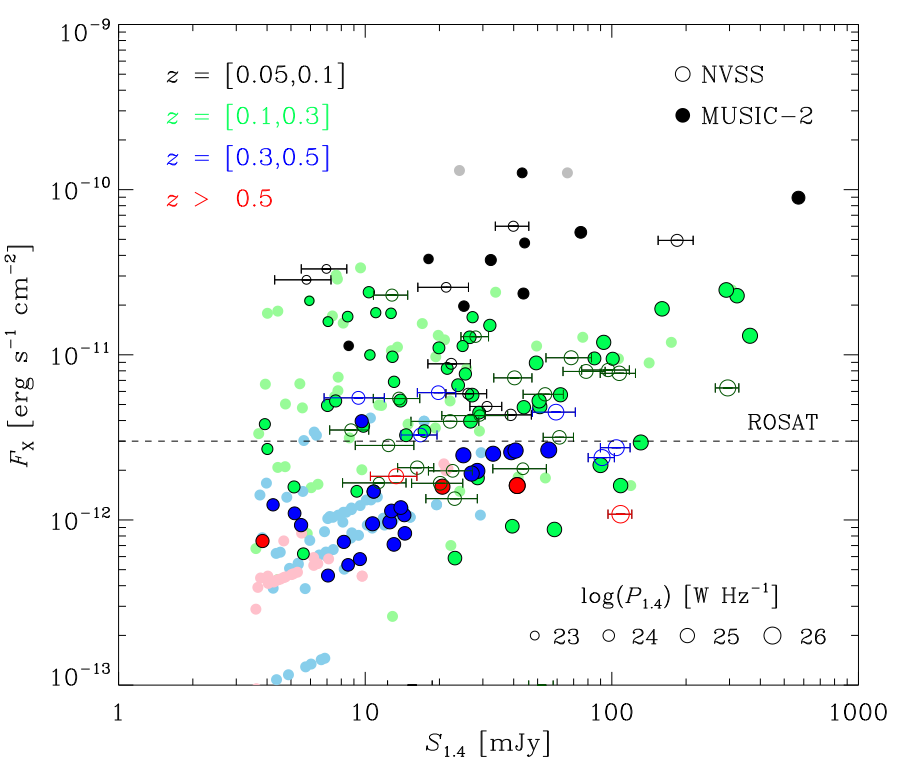

Figure 14. X-ray flux, $F_{X}$, versus total radio flux, $S_{1.4}$, for clusters hosting relics. Shown are the NVSS clusters (empty circles) and those belonging to the 'elongated' (solid circles) and 'smallroundish' (light solid circles) mock relic samples. Symbol sizes for the NVSS and 'elongated' samples are scaled according to radio power. In all cases, redshift bins are indicated using different colours. The ROSAT flux limit is included here for reference (dashed line).

structures. In particular, the median orientation angle for the 'elongated' and NVSS samples is $\langle\phi\rangle \simeq 69^{\circ}$. In the same line, mean shape values of both distributions are virtually identical with $\langle s\rangle \simeq 0.31$.

These results can be interpreted in a simple way: the vast majority of the elongated structures are most likely produced by mergers with low impact parameters (i.e., in head-on collisions or close). In this case, pseudo-spherical surfaces of shocked material sweeping the ICM are generated. When seeing edge-on, these surfaces are observed as arc-like radio features perpendicular to the line joining the relic and cluster centres. This is what one would expect for shock fronts travelling outwards the merger's axis direction.

Fig. 17] shows the LLS versus the projected distance,

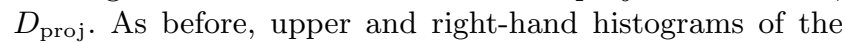
corresponding quantities are also presented. In MUSIC- 2 we obtain relics spanning similar sizes and projected locations within galaxy clusters as those found in the NVSS sample. However, the mock relics tend to be smaller and closer to the cluster centre, which is more evident in the case of the 'small-roundish' sample. For the 'elongated' and NVSS samples the mean LLS values are 0.7 and $0.82 \mathrm{Mpc}$. In the case of projected distances differences are higher with mean values of 0.71 and $1.07 \mathrm{Mpc}$, respectively. These discrepancies become particularly striking when considering distant clusters at $z \gtrsim 0.3$, which could originate from the existence of too compact clusters at higher redshifts in our simulations. From the histograms of Fig. 17, it can be seen that the two simulated distributions clearly peak towards smaller values in comparison to the NVSS. A possible explanation of the

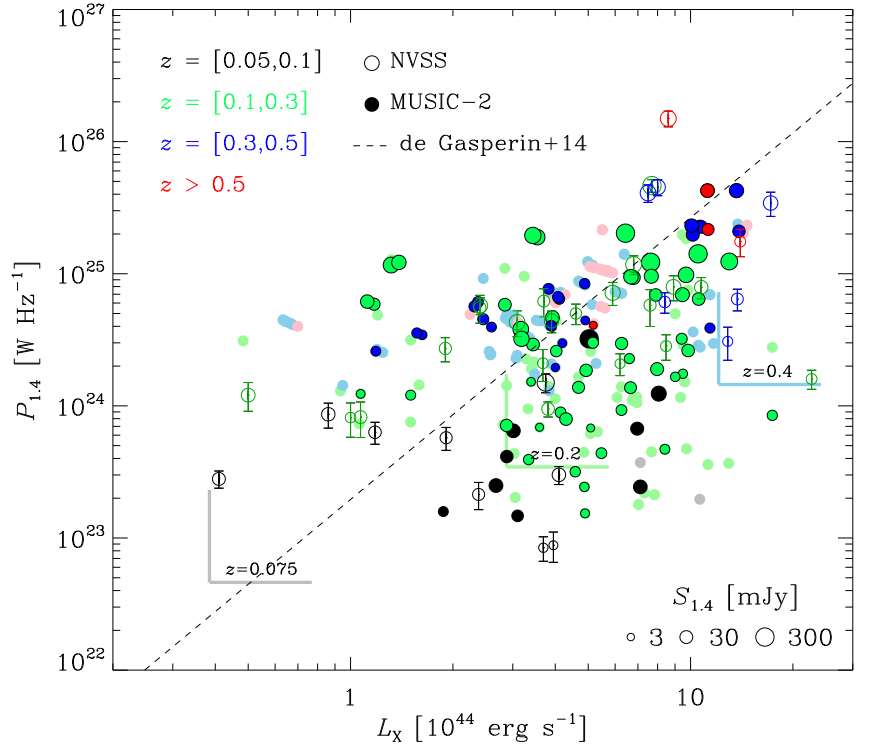

Figure 15. Radio power, $P_{1.4}$, versus X-ray luminosity, $L_{\mathrm{X}}$, for clusters hosting relics. Shown are the NVSS clusters (empty circles) and those belonging to the 'elongated' (solid circles) and 'small-roundish' (light solid circles) mock relic samples. Symbol sizes for the NVSS and 'elongated' samples are scaled according to radio flux. In all cases, redshift bins are indicated using different colours. The radio and X-ray luminosity thresholds associated with the minimum radio flux imposed to our samples, $S_{1.4, \min }=8 \times \sigma_{\mathrm{NVSS}}$ (horizontal solid lines), and those corresponding to the ROSAT flux limit (vertical solid lines) for different redshifts, are also shown. For reference, we also include the de Gasperin et al. (2014) relation after rescaling cluster masses to the virial radius and transform to X-ray luminosity using Eq. 7 (dashed line).

latter may be related to the lack of ICM heating in our simulations which could artificially increase the Mach number of the shocks. However, further work is needed to decide on this matter (see Section 5.2.

Our findings show that radio relics statistics are well suited to investigate the evolution of the ICM, because the Mach number is sensitive to the ICM temperature. We want to stress that, observationally, relics are typically searched as extended diffuse emission in the periphery of galaxy clusters. Therefore, there might also be a classification bias in the NVSS sample, causing that more centrally located objects with the same physical origin as large peripheral relics, are either not detected or not classified as such.

\subsection{Relic abundance}

So far, we have not addressed a crucial question, namely, if the MUSIC-2 simulation reproduces the number of relics found in NVSS. The reason to postpone this discussion is that we needed to identify a region of the parameter space where the simulated and observed cluster samples can be compared. The NVSS relic sample is essentially flux limited with clusters spanning almost two decades in X-ray luminosities (see Fig. 15). On the other hand, the MUSIC-2 cluster sample is rather massive, hosting unambiguosly detected 


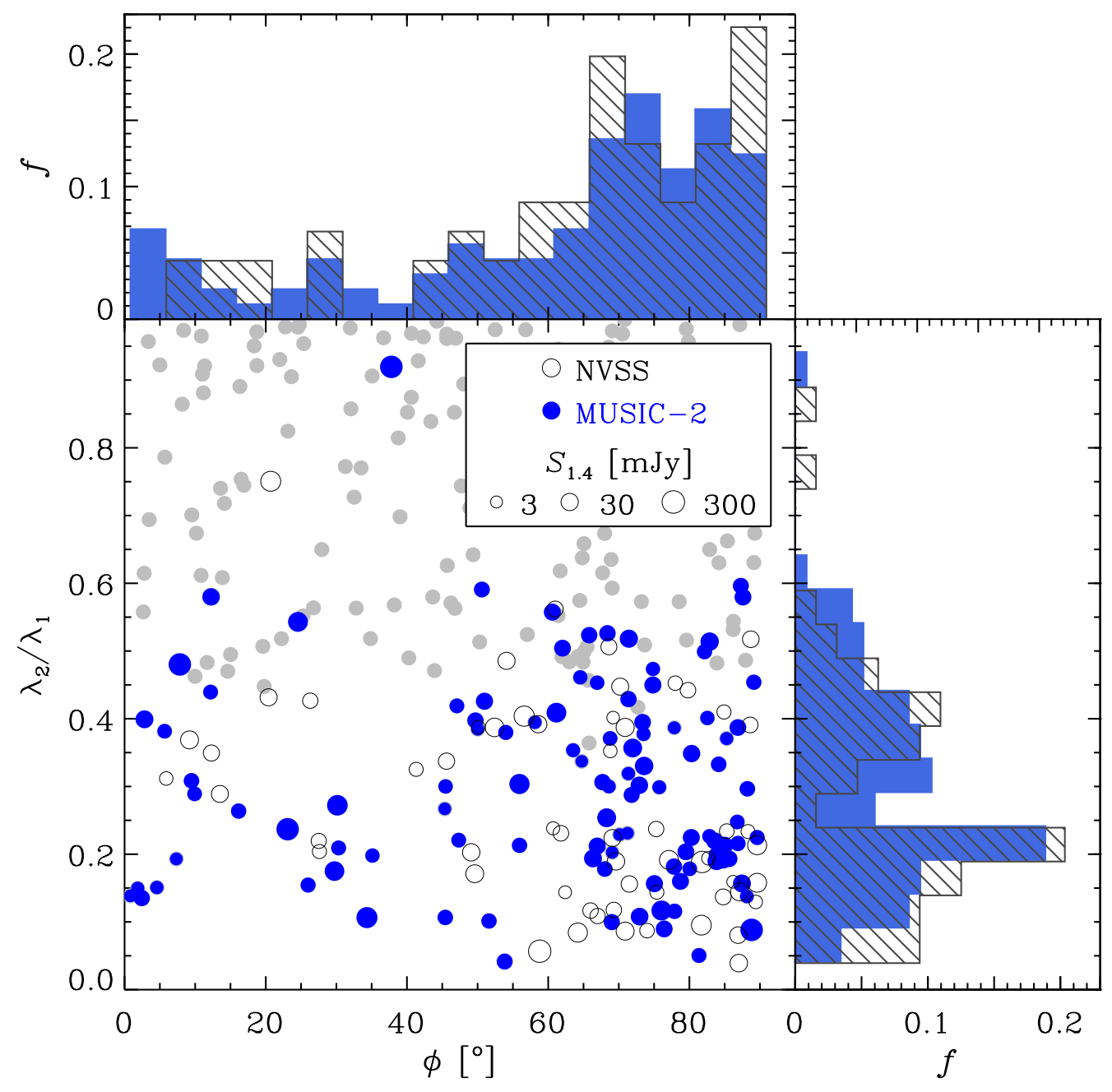

Figure 16. Shape, $s \equiv \lambda_{2} / \lambda_{1}$, versus orientation angle within the cluster, $\phi$, for the relic samples. Shown are the NVSS islands (empty circles) and the 'elongated' (blue solid circles) and 'small-roundish' (light solid circles) mock relic samples. Symbol sizes for the NVSS and 'elongated' samples are scaled according to radio flux. The upper and right-hand histograms show the comparison between the NVSS (dashed histogram) and 'elongated' (blue histogram) samples for the orientation angle and shape parameter, respectively.

radio islands well below $10 \mathrm{mJy}$. The comparison between the NVSS and 'elongated' mock cluster samples extracted from the MUSIC-2 simulations is shown in Fig. 5, where we plot the cumulative number counts with flux density larger than $S_{1.4}$. As discussed in Section 3.2, the MUSIC-2 cluster sample is only complete for $L_{\mathrm{X}} \gtrsim 3 \times 10^{44} \mathrm{erg} \mathrm{s}^{-1}$, therefore, we exclude all NVSS clusters below this limit from the plot (black dashed curve). We then take into account that NVSS covers only $82 \%$ of the sky and correct for incompleteness (red dashed curve). Evidently, in MUSIC-2 we find significantly more relics than in observations. We emphasise that, in order to properly compare to NVSS, we have already excluded the 'small-roundish' islands found in some of the MUSIC-2 clusters. We speculate that the lower abundance of relics in NVSS reflects a flux-dependent completeness. In fact, towards lower flux densities the discrepancy increases. At $S_{1.4} \lesssim 10 \mathrm{mJy}$, the MUSIC-2 predicts about two times more relics than found in NVSS (see Fig. 18). Interestingly, if we apply the ROSAT limit to the 'elongated' sample we found that the number of clusters hosting relics in the two samples roughly match. This suggests a natural explanation for the excess of objects in the 'elongated' sample: a significant fraction of the observed relics have simply not been detected or classified as their host clusters remain unidentified. We note, however, that we are dealing with small number statistics and only future X-ray and radio surveys can confirm or reject this hypothesis.

We are now in position to further discuss the results of Fig. 6, where we plotted the cumulative number of clusters hosting relics versus $L_{\mathrm{X}}$. As before, results corresponding to the 'elongated' mock cluster samples extracted from the MUSIC-2 simulations are indicated by shaded regions. To properly compare with observations, we have excluded all mock clusters hosting relics with a flux below the NVSS threshold, which, we set equal to $7 \mathrm{mJy}$ (see solid line in Fig. 5). The NVSS and its all-sky corrected distributions 


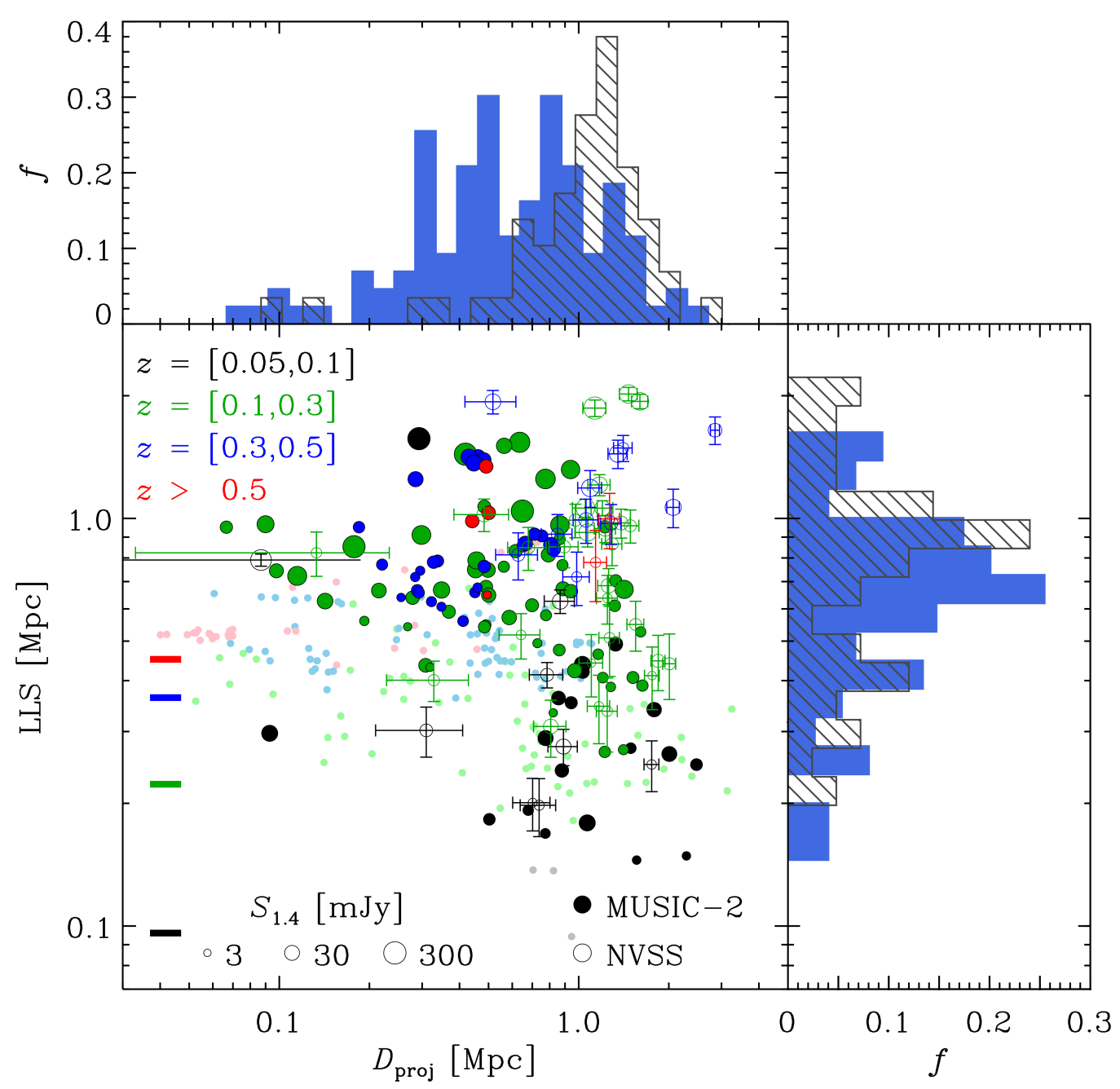

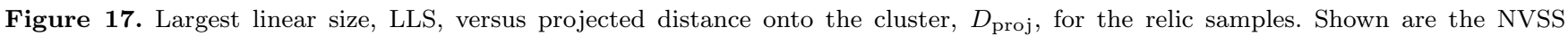
clusters (empty circles) and those belonging to the 'elongated' (solid circles) and 'small-roundish' (light solid circles) mock relic samples. Symbol sizes for the NVSS and 'elongated' samples are scaled according to radio flux. In all cases, redshift bins are indicated using different colours. The upper and right-hand histograms show the comparison between the NVSS (dashed histogram) and 'elongated' (blue histogram) samples for $D_{\text {proj }}$ and LLS, respectively.

are also shown (solid and red dashed lines, respectively). As before, the mock cluster counts are larger than for observations by roughly a factor of two (solid circles) and they nicely match if we exclude clusters below the ROSAT detection limit (open circles). Interestingly, a few NVSS clusters have higher X-ray luminosities $\left(L_{\mathrm{X}} \gtrsim 10^{45} \mathrm{erg} \mathrm{s}^{-1}\right)$ than for the 'elongated' sample. Two effects can contribute to this. We have adopted a general mass-luminosity relation to assign X-ray luminosities to the simulated clusters. During a merger event, however, the cluster X-ray luminosity can significantly deviate from the scaling relation and the system may boost its X-ray emission. Thus, we possibly slightly underestimate the X-ray luminosities. Moreover, due to the finite size of the simulation volume, we miss very massive clusters. This is particularly important at higher redshifts, where the observable volume is much larger than the simulated one.
As a final remark, we note that the abundance of relics in our model is essentially controlled by the magnetic field and the efficiency parameter. For the adopted magnetic field model, we have deliberately chosen an efficiency parameter of $\xi_{\mathrm{e}}=5 \times 10^{-5}$ to match the NVSS cluster counts at $S_{1.4}=$ $100 \mathrm{mJy}$, a flux for which is assumed that almost all relics are known. As shown above, the resulting sample of mock relics reproduce the NVSS observed correlations reasonably well. The most significant mismatch in the relic properties has been found for the sizes and projected distances of relics in distant clusters at $z \gtrsim 0.3$. Clearly, a different magnetic field model -assuming another efficiency parameter- may have an impact on the size and location of relics. It is beyond the scope of the present work to explore this in detail. 


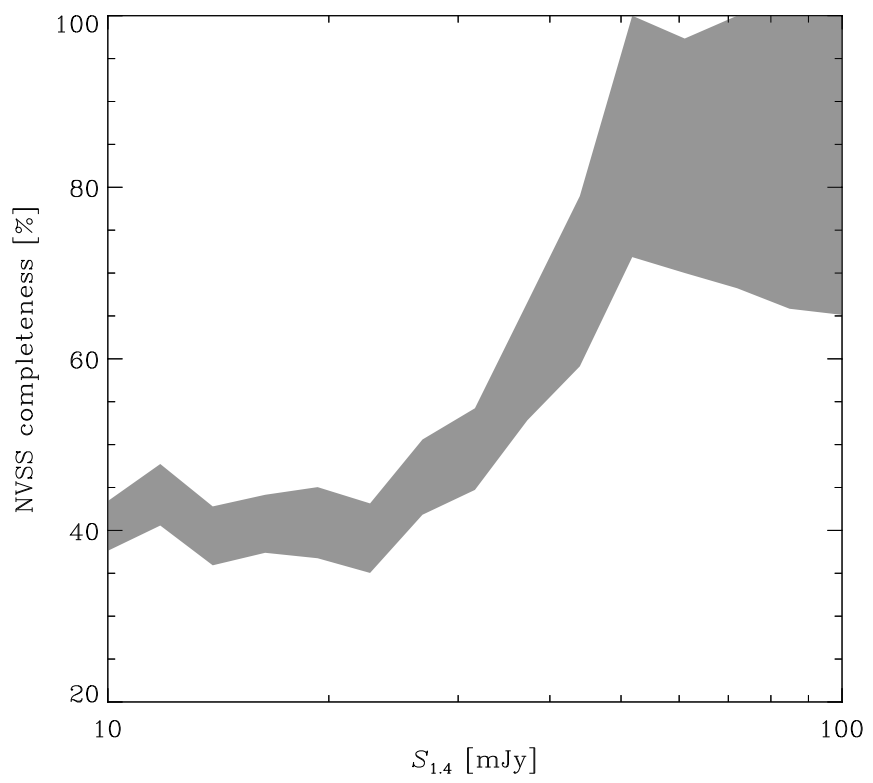

Figure 18. Estimated completeness of the NVSS relic sample derived by the ratio between the cumulative number of NVSS to 'elongated' cluster samples (shaded region). The NVSS cluster number is scaled to full sky adopting a sky coverage of $82 \%$.

\subsection{Radio luminosity versus cluster mass}

The strong correlation between radio power and X-ray luminosity may also indicate that more massive clusters could generate more luminous radio relics de Gasperin et al. 2014). We explore this scaling in Fig. 19. which shows total radio power versus cluster mass in the MUSIC-2 'elongated' sample. As before, we also include the scaled best-fit relation of de Gasperin et al. (2014) for comparison. The detection threshold (i.e., the radio flux limit) increases with redshift, as reflected by the colour-coding of symbols. In particular, the interval $z \in[0.1,0.3]$ covers a fairly small redshift range while having enough statistics to allow us investigate the existence of a luminosity-mass correlation free of any Malmquist bias effect. It is evident that the most massive clusters in the sample host the most luminous relics. Still, the scatter in radio luminosity is large. Therefore, less massive clusters can eventually produce similarly bright relics as those with larger mass, see e.g. luminosities corresponding to $M_{\text {vir }} \sim 10^{14.9}$ and $10^{15.2} \mathrm{M}_{\odot}$ in Fig. 19 .

As mentioned above, it is evident that the radio power normalization of relics in more distant clusters is higher. According to this, a correlation between radio luminosity and cluster mass would imply that only the bright relics in massive clusters at high redshift are detected. As a result, the detection threshold could amplify the observed correlation. The mass range covered in the MUSIC-2 simulations is too small to derive an average luminosity-mass correlation for small redshift intervals, i.e. free of Malmquist bias. We speculate, however, that the maximum radio luminosity at a given mass actually increases with cluster mass, but probably less steeply than found for samples affected by the latter.

\section{DISCUSSION}

\subsection{The 'small-roundish' sample}

The small-roundish sample is, basically, exclusively found in MUSIC-2 with islands having the following properties: (i) they are small and roundish with $s / \mathrm{LAS}>0.17$ (see Fig. 9); (ii) they tend to be found at higher redshifts, hence, predominantly residing in clusters with low X-ray flux; (iii) they show median values of LLS and projected distance of about 500 and $400 \mathrm{kpc}$ respectively, and (iv) the median radio luminosities of these objects are higher than in observations.

In the NVSS sample there are only a few objects in the 'small-roundish' regime, namely, the recently discovered gischt double candidate in CIZA J0107 Randall et al. 2016); the north-western edge emission in Abell 1682 (Venturi et al. 2013); the southern steep-spectrum feature in the 'Toothbrush' cluster (van Weeren et al. 2016), and the recently discovered gischt candidate in PLCK G004 (Sifón et al. 2014). All of these objects are also rather small in size and the islands in Abell 1682, the 'Toothbrush' cluster, and PLCK G004 do not show the typical relic morphology. This may indicate that the identification of these kind of objects in many NVSS clusters is not free of difficulties. Therefore, it is possible that many of them are still not yet discovered. In particular, we found about 100 of these 'small-roundish' islands in the MUSIC-2 simulations within the context of our model.

\subsection{Numerical scheme and ICM physics}

The abundance and location of shocks fronts is affected by the particular numerical scheme used. For instance, Vazza et al. (2011) has shown that Eulerian codes tend to have a smaller fraction of shock fronts with Mach numbers of 2-4 in low-entropy gas compared to standard SPH simulations. This suggest that an Eulerian numerical scheme would result in less shock fronts close to the cluster centre. However, recent SPH implementations have been shown to better treat fluid mixing thus alleviating these problems (e.g., Hopkins 2013).

The physical state of the ICM is also affected by galactic and AGN feedback, which is not included in the simulation we use here. It has been shown that, in average, the feedback increases the ICM temperature (see e.g., Sembolini et al. 2016). This is particularly important for the inner parts of the cluster. A higher ICM temperature will result in lower Mach numbers and, hence, make central radio relics less likely.

Therefore, the two major discrepancies found between the MUSIC-2 and the NVSS sample, i.e. too many smallroundish objects and the presence of high-redshift elongated relics with somewhat smaller projected distances and sizes, might be partly spurious due to the numerical scheme and missing physics of the simulation. However, there are also several additional ingredients affecting the matching between the two samples: the uncertain completeness of the NVSS, the magnetic field distribution in the ICM and the dependence of the radio luminosity on shock parameters. We have to leave to future studies the assessment of the impact of all these factors in the comparison between observations and simulations. 


\subsection{On the NVSS completeness}

Several fairly bright radio relics have not been discovered until recently, e.g. the relics in PLCK G287 (Bagchi et al.|2011) and PSZ1 G108 (de Gasperin et al. 2015) with a flux density of $58 \mathrm{mJy}$ and $113 \mathrm{mJy}$, respectively. These objects are well visible in NVSS as extended sources. Evidently, since the clusters were not known prior to PLANCK observations, the emission was not classified as radio gischt. This suggests that there might be unclassified single or double gischt objects already visible in NVSS.

The comparison between the NVSS and MUSIC-2 samples indicates that only about $40 \%$ of the extended relics with a total flux smaller than $10 \mathrm{mJy}$ have been discovered in X-ray luminous clusters with $L_{X} \gtrsim 3 \times 10^{44} \mathrm{erg} \mathrm{s}^{-1}$. For example, the double gischt in Abell 1240 is rather difficult to identify in NVSS because each relic has a flux of about $5 \mathrm{mJy}$ (see Fig. 11). The flux of the entire gischt emission is distributed over at least 10 beams. Only part of the relic is recovered by the $2 \times \sigma_{\text {NVSS }}$ contours. This illustrates the fact that systematic searches for radio relics at $10 \mathrm{mJy}$ and below in NVSS were incomplete.

\subsection{On the gischtlet class}

In Section 2.1 we introduced a class of radio relics with the same physical origin of gischts but with a different morphology: the gischtlets. In contrast to classical gischts, these objects are rather small, are located close to the cluster centre, and may show complex morphologies. Reference cases might be the ridges found in Abell 1682 (Venturi et al. 2013) and the large but central 'chair-shaped' filament in MACS 0117 (Bonafede et al. 2009, van Weeren et al. 2009b). In both cases, the morphology does not obviously agree with that of large merger shock fronts travelling outwards.

Fig. 7 shows a few objects with rather unusual morphology that could lie within this classification, e.g. the relic in cluster ' $\mathrm{B}$ ' which is rather close to the cluster centre and that of cluster ' $\mathrm{D}$ ' which is strangely not aligned with the apparent merger axis. Hence, a non-negligible fraction of the objects found in the MUSIC-2 sample does not resemble a classical large gischt caused by a binary merger. This again illustrates that the classification based on morphology may cause that some relics are not recognized as a result of their unusual appearance.

\subsection{Upcoming surveys}

Our approach is applied to the NVSS sample of relics, yet it is not tied to any specific survey. There are several upcoming radio wide-field surveys, each yielding a large increase in both sensitivity and resolution compared to NVSS.

For instance, APERTIF-Wodan and ASKAP-EMU (Norris et al. 2011) have similar survey specifications and are highly complementary because of their low-intersecting sky coverage. These surveys are going to probe the continuum radio sky at frequencies of $1100-1400 \mathrm{MHz}$. With an expected survey depth of $10 \mathrm{mJy}$ and a 10 arcsec resolution, the sensitivity for extended structures will exceed NVSS by more than a factor of 10 .

The LOFAR radio telescope is going to probe the northern radio sky at $120-168 \mathrm{MHz}$ in three different stages or

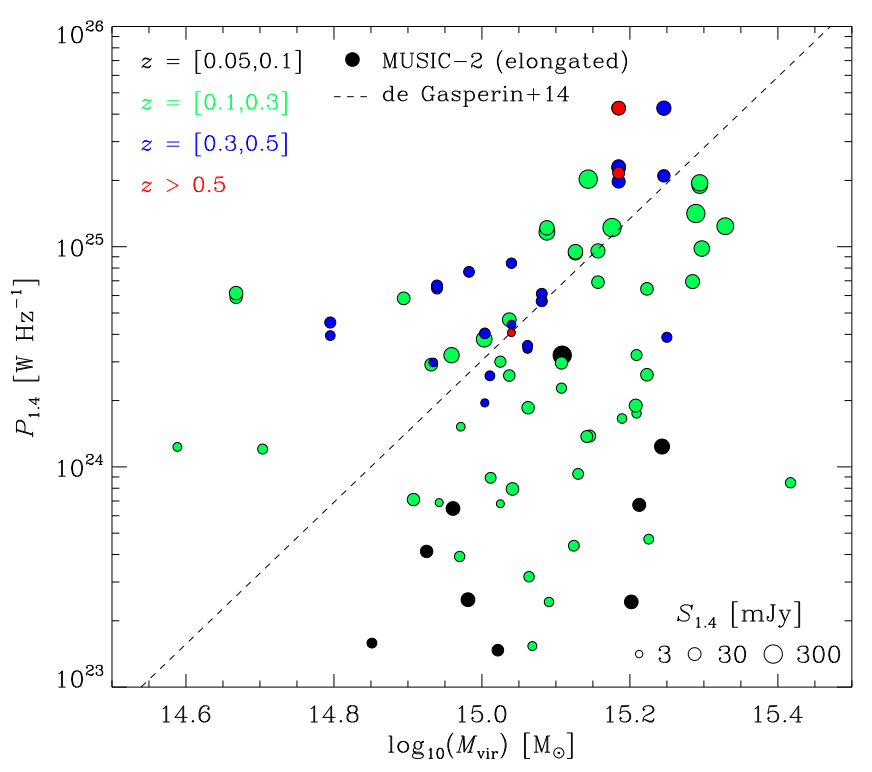

Figure 19. Radio power, $P_{1.4}$, versus cluster virial mass, $M_{\text {vir }}$, for the 'elongated' mock sample (solid circles). Symbol sizes are scaled according to radio flux. Redshift bins are indicated using different colours. For reference, we also include the de Gasperin et al. (2014) relation after rescaling cluster masses to the virial radius (dashed line).

'tiers'. Tier I will be the most shallow but largest survey with 5 arcsec resolution and $0.1 \mathrm{mJy}$ noise per beam. Sensitivity for both point sources and extended structures will exceed that of the GMRT $150 \mathrm{MHz}$ All-sky Radio Survey (Intema et al. 2016) by, at least, an order of magnitude, translating into 10 times better sensitivity for extended objects with a spectral index of $\alpha=-1$.

These surveys are expected to significantly increase the number of known radio relics. Ultimately, the relic sample will be expanded towards lower surface brightnesses. The combination of all these new observations, together with more sophisticated large-volume cosmological simulations, will allow for a more thorough comparison between models and reality. Additionally, synergies with panchromatic surveys (e.g., X-ray; SZ) and optical follow-up observations, will be necessary to identify galaxy clusters and determine their redshift.

In this work, we explored one scenario to model the non-thermal radio emission of radio relics. However, our approach is well suited to discriminate between different models, not only DSA, but also more complex acceleration mechanisms. We leave this for future research.

\section{SUMMARY}

The origin of radio relics is quite enigmatic. There is ample evidence indicating that relics are located at shock fronts originated in merging galaxy clusters. However, it is not clear if all merger shocks are, in turn, able to produce radio relics. To shed light on this issue, we compared the flux, size, morphology, orientation and location of mock relic structures linked to simulated merger shocks with an homoge- 
neous sample of known radio relics. Moreover, the simulated host galaxy clusters were used to further study the well-known correlation between radio power and X-ray luminosity and its most recent version involving cluster mass (de Gasperin et al. 2014).

To perform the relic comparison, we compiled a comprehensive list of radio relics and candidates based on previous collections (Nuza et al. 2012, Feretti et al. 2012, Yuan et al. 2015) and recently published relics. We distinguished radio phoenixes and gischts according to the classification given in literature. In this work, a radio gischt is assumed to originate from cosmological merger shock fronts.

For all possible relics in our list, we determined their properties in NVSS images. To define the boundary of radio islands, we used $2 \times \sigma_{\text {NVSS }}$ contours, where $\sigma_{\text {NVSS }}$ is the sensitivity limit of the NVSS. For each island, we measured flux density, LAS, LLS, solid angle, shape based on image moments and orientation with respect to the cluster. In total, we identified 59 islands in 39 galaxy clusters. A crucial aspect of our approach is to determine relic parameters in the most homogeneous possible way.

To model relics, we used 282 resimulated galaxy cluster regions extracted from a cosmological comoving volume of $1 h^{-3} \mathrm{Gpc}^{3}$ comprising the so-called MUSIC-2 simulations. The hydrodynamical evolution of the clusters has been simulated using an SPH code including non-radiative physics only. For our purpose, it is mandatory to have as many massive clusters as possible from a large cosmological volume. Still, every cluster has to be simulated with sufficient resolution to properly describe the shock fronts. We applied the shock finder presented by Hoeft et al. (2008) to each snapshot in post-processing to find the shock fronts and to estimate their associated radio emission.

For lighting up the shocks, we used the radio-emission model and parameters given by Nuza et al. (2012). In particular, we adopted the 'Bonafede' scaling for estimating the magnetic field strength as a function of local gas density and $\xi_{\mathrm{e}}=5 \times 10^{-5}$ for the efficiency parameter. The latter has been chosen to restore the number of bright relics found in the NVSS sample.

For each snapshot, we carried out mock cluster observations adopting NVSS specifications and determined regions of radio emission in the same way as for NVSS images. To account for the fact that the observable volume of the universe increases with redshift, we computed a probability quantifying how often any given simulated cluster should be considered part of the sample. To avoid duplicating relic parameters, we randomly rotated each cluster every time it enters more than once into the mock sample.

Our main findings can be summarized as follows:

- For both the NVSS and the mock relic samples, we found that the shape, $s$, and LAS (anti-)correlate in a similar way. In particular, mean shape values for these two samples are virtually identical with $\langle s\rangle \simeq 0.31$. This shows that our simulations produce relics with a similar appearance to observations. Interestingly, our mock relic sample does not show the presence of a significant population of large and roundish relics. This suggests that current radio halo samples, which comprise diffuse radio emssion following the cluster X-ray distribution, are not significantly contaminated by radio relics seen face-on.

- In the mock relic sample, we found many 'smallroundish' islands fulfilling the condition $s / \mathrm{LAS}>0.17$. Only very few radio islands with similar properties are found in the NVSS sample. This 'small-roundish' sample comprises some compact features but also more extended relics in distant clusters. We speculate that compact features in clusters are spurious being, most likely, the result of a combination of the simulation technique and the lack of energy feedback in our simulations. However, extended objects at high redshift may reflect realistic merger shocks which are not detected in NVSS since they have a small LAS, they appear almost roundish, and most importantly, their host clusters have not yet been discovered. The relic candidate found in CIZA J0107 is an example of a 'small-roundish' double relic in NVSS. The MUSIC-2 mock relic sample suggests that many more similar, but not yet identified, objects should be present in NVSS.

- The mock 'elongated' sample nicely reproduces the correlation between radio power and LLS. We argue that this correlation is equivalent to the finding that the average surface brightness of all relics is located in a fairly narrow range. This small range may originate from the fact that deeper observations usually reveal more regions with low surface brightness thus increasing the relic extension and balancing the brightening.

- Consistent with previous studies, we found that relics in the NVSS sample tend to be tangentially oriented with respect to the axis joining the relic and cluster centres. This suggests that the vast majority of structures found in NVSS are most likely produced in head-on cluster collisions or close. We confirm this result using our simulations. In particular, elongated structures both in NVSS and the mock relic sample are typically clustered at $(s, \phi)=\left(\lesssim 0.5, \gtrsim 60^{\circ}\right)$ in the shape-orientation plane. For the 'elongated' and NVSS samples, the median orientation angle turns out to be $\phi \simeq 69^{\circ}$. As expected, this signal is essentially lost when considering more roundish objects also present in our simulations.

- We found that the mock 'elongated' relic sample spans similar LLS and projected distance $\left(D_{\text {proj }}\right)$ values than NVSS. However, in average, 'elongated' mock relics tend to have somewhat smaller sizes and to be located closer to the cluster centre in comparison to observations (i.e., $\langle\mathrm{LLS}\rangle=0.7,0.82 \mathrm{Mpc}$ and $\left\langle D_{\text {proj }}\right\rangle=0.71,1.07 \mathrm{Mpc}$ for the 'elongated' and NVSS samples, respectively). Moreover, these discrepancies become larger when considering more distant relics at $z \gtrsim 0.3$. A possible explanation to this might be given by the lack of supernova and AGN feedback in our simulations. Energy feedback would heat the ICM and increase the sound speed in the central parts of the simulated galaxy clusters. Nonetheless, further work is needed to decide on this issue.

- We found that the well-known radio power versus $\mathrm{X}$-ray cluster luminosity correlation is to a large extent determined by the flux detection threshold both in radio and X-ray. Similarly, the related radio power versus cluster 
mass correlation recently proposed is artificially strengthten by the flux limits: at higher redshifts, bright relics in massive clusters are preferentially detected. Since scatter in these correlations is large, our simulations suggest that the peak radio luminosity actually scales with mass but in a less-steep way than found for Malmquist bias-affected samples.

- We estimated a flux-dependent completeness of the NVSS relic sample of about $40 \%$ at $10 \mathrm{mJy}$. We note that this might be partly spurious since the simulation may overpredict low-luminosity relics. However, we argue that the derived efficiency is plausible given the fact that $10 \mathrm{mJy}$ relics are difficult to discover in NVSS. Furthermore, if we apply the ROSAT X-ray flux limit to the 'elongated' mock relic sample - which is basically the limit shown by NVSS clusters- we roughly reproduce the observed cluster number counts. If it is true that the discovery of radio relics is currently limited by the number of available X-ray clusters used to identify them, this would provide additional support to our conclusion on the completeness of the NVSS sample.

Overall the mock 'elongated' gischt sample reproduces the NVSS-derived relic properties reasonably well, specially regarding shape, orientation, flux, radio luminosity and cluster X-ray luminosity distributions, when taking into account all known biases of the samples. This suggests that the morphology and abundance of merger shocks in a cosmological framework is, in principle, enough to explain radio relics. These findings may rule out the scenario where merger shocks are only partly illuminated by pre-existing radio plasma. If such a plasma was necessary to explain all radio relics, it would have to be distributed rather homogeneously in all galaxy clusters.

Clearly, upcoming deeper radio surveys, such as LOFAR-Tier-1, EMU or Apertif, will significantly enhance the sample of known radio relics. Exploiting this data with the aid of more advanced theoretical models and simulations will help us to better understand the elusive nature of these objects.

\section{ACKNOWLEDGEMENTS}

The MUSIC simulations have been performed in the MareNostrum supercomputer at the Barcelona Supercomputing Centre thanks to the computing time awarded by the Red Española de Supercomputación. SEN, JG and MH acknowledge support by the Deutsche Forschungsgemeinschaft under grant NU 332/2-1 and research group FOR 1254. GY acknowledges support from MINECO/FEDER under grants AYA2012-31101 and AYA2015-63810-P.

\section{REFERENCES}

Akamatsu H., van Weeren R. J., Ogrean G. A., Kawahara H., Stroe A., Sobral D., Hoeft M., Röttgering H., Brüggen M., Kaastra J. S., 2015, A\&A, 582, A87

Bagchi J., Durret F., Neto G. B. L., Paul S., 2006, Science, 314,791
Bagchi J., Sirothia S. K., Werner N., Pandge M. B., Kantharia N. G., Ishwara-Chandra C. H., Gopal-Krishna Paul S., Joshi S., 2011, ApJL, 736, L8

Basu K., Vazza F., Erler J., Sommer M., 2016, A\&A, 591, A142

Becker R. H., White R. L., Helfand D. J., 1995, ApJ, 450, 559

Bertin E., Arnouts S., 1996, A\&A S, 117, 393

Blandford R., Eichler D., 1987, PhysRep, 154, 1

Böhringer H., Chon G., Collins C. A., 2014, A\&A, 570, A31

Böhringer H., Voges W., Huchra J. P., McLean B., Giacconi

R., Rosati P., Burg R., Mader J., Schuecker P., Simiç D., Komossa S., Reiprich T. H., Retzlaff J., Trümper J., 2000, ApJS, 129, 435

Bonafede A., Brüggen M., van Weeren R., Vazza F., Giovannini G., Ebeling H., Edge A. C., Hoeft M., Klein U., 2012, MNRAS, 426, 40

Bonafede A., Feretti L., Giovannini G., Govoni F., Murgia M., Taylor G. B., Ebeling H., Allen S., Gentile G., Pihlström Y., 2009, A\&A, 503, 707

Bonafede A., Feretti L., Murgia M., Govoni F., Giovannini G., Dallacasa D., Dolag K., Taylor G. B., 2010, A\&A, 513, $\mathrm{A} 30+$

Bonafede A., Giovannini G., Feretti L., Govoni F., Murgia M., 2009, A\&A, 494, 429

Bonafede A., Intema H., Brüggen M., Vazza F., Basu K., Sommer M., Ebeling H., de Gasperin F., Röttgering H. J. A., van Weeren R. J., Cassano R., 2015, MNRAS, 454, 3391

Bonafede A., Vazza F., Brüggen M., Murgia M., Govoni F., Feretti L., Giovannini G., Ogrean G., 2013, MNRAS, 433, 3208

Boschin W., Barrena R., Girardi M., Spolaor M., 2008, A\&A, 487, 33

Botteon A., Gastaldello F., Brunetti G., Dallacasa D., 2016, ArXiv e-prints [astro-ph:1604.07823]

Brown S., Duesterhoeft J., Rudnick L., 2011, ApJL, 727, L25+

Cantwell T. M., Scaife A. M. M., Oozeer N., Wen Z. L., Han J. L., 2016, MNRAS, 458, 1803

Caprioli D., 2012, JCAP, 7, 038

Clarke T. E., Ensslin T. A., 2006, AJ, 131, 2900

Cohen A. S., Clarke T. E., 2011, AJ, 141, 149

Condon J. J., Cotton W. D., Greisen E. W., Yin Q. F., Perley R. A., Taylor G. B., Broderick J. J., 1998, AJ, 115, 1693

de Gasperin F., Intema H. T., Ridl J., Salvato M., van Weeren R., Bonafede A., Greiner J., Cassano R., Bruggen M., 2016, ArXiv e-prints [astro-ph:1609.08339]

de Gasperin F., Intema H. T., van Weeren R. J., Dawson W. A., Golovich N., Wittman D., Bonafede A., Brüggen M., 2015, MNRAS, 453, 3483

de Gasperin F., Ogrean G. A., van Weeren R. J., Dawson W. A., Brüggen M., Bonafede A., Simionescu A., 2015, MNRAS, 448, 2197

de Gasperin F., van Weeren R. J., Brüggen M., Vazza F., Bonafede A., Intema H. T., 2014, MNRAS, 444, 3130

Dolag K., Schindler S., Govoni F., Feretti L., 2001, A\&A, 378,777

Donnert J. M. F., Stroe A., Brunetti G., Hoang D., Roettgering H., 2016, MNRAS, 462, 2014

Drury L., 1983, SSRv, 36, 57 
Duffy A. R., Schaye J., Kay S. T., Dalla Vecchia C., 2008, MNRAS, 390, L64

Ebeling H., Barrett E., Donovan D., Ma C.-J., Edge A. C., van Speybroeck L., 2007, ApJL, 661, L33

Ebeling H., Edge A. C., Allen S. W., Crawford C. S., Fabian A. C., Huchra J. P., 2000, MNRAS, 318, 333

Ebeling H., Edge A. C., Bohringer H., Allen S. W., Crawford C. S., Fabian A. C., Voges W., Huchra J. P., 1998, MNRAS, 301, 881

Ebeling H., Mullis C. R., Tully R. B., 2002, ApJ, 580, 774 Ebeling H., Voges W., Bohringer H., Edge A. C., Huchra J. P., Briel U. G., 1996, MNRAS, 281, 799

Ensslin T. A., Biermann P. L., Klein U., Kohle S., 1998, A\&A, 332, 395

Enßlin T. A., Gopal-Krishna 2001, A\&A, 366, 26

Farnsworth D., Rudnick L., Brown S., Brunetti G., 2013, ApJ, 779, 189

Feretti L., Bacchi M., Slee O. B., Giovannini G., Govoni F., Andernach H., Tsarevsky G., 2006, MNRAS, 368, 544 Feretti L., Fusco-Femiano R., Giovannini G., Govoni F., 2001, A\&A, 373, 106

Feretti L., Giovannini G., 1996, in Ekers R. D., Fanti C., Padrielli L., eds, Extragalactic Radio Sources Vol. 175 of IAU Symposium, Diffuse Cluster Radio Sources (Review). p. 333

Feretti L., Giovannini G., Govoni F., Murgia M., 2012, A\&A Rev., 20, 54

Feretti L., Schuecker P., Böhringer H., Govoni F., Giovannini G., 2005, A\&A, 444, 157

Finoguenov A., Sarazin C. L., Nakazawa K., Wik D. R., Clarke T. E., 2010, ApJ, 715, 1143

Giacintucci S., Venturi T., Bardelli S., Brunetti G., Cassano R., Dallacasa D., 2006, NewA, 11, 437

Giacintucci S., Venturi T., Cassano R., Dallacasa D., Brunetti G., 2009, ApJL, 704, L54

Giovannini G., Bonafede A., Feretti L., Govoni F., Murgia M., 2010, A\&A, 511, L5+

Giovannini G., Bonafede A., Feretti L., Govoni F., Murgia M., Ferrari F., Monti G., 2009, A\&A, 507, 1257

Giovannini G., Feretti L., 2000, NewA, 5, 335

Giovannini G., Tordi M., Feretti L., 1999, NewA, 4, 141

Govoni F., Feretti L., Giovannini G., Böhringer H., Reiprich T. H., Murgia M., 2001, A\&A, 376, 803

Govoni F., Ferrari C., Feretti L., Vacca V., Murgia M., Giovannini G., Perley R., Benoist C., 2012, A\&A, 545, A74

Govoni F., Murgia M., Giovannini G., Vacca V., Bonafede A., 2011, A\&A, 529, A69

Guo X., Sironi L., Narayan R., 2014a, ApJ, 794, 153

Guo X., Sironi L., Narayan R., 2014b, ApJ, 797, 47

Hoeft M., Brüggen M., 2007, MNRAS, 375, 77

Hoeft M., Brüggen M., Yepes G., Gottlöber S., Schwope A., 2008, MNRAS, 391, 1511

Hoeft M., Nuza S. E., Gottlöber S., van Weeren R. J., Röttgering H. J. A., Brüggen M., 2011, Journal of Astrophysics and Astronomy, 32, 509

Hong S. E., Kang H., Ryu D., 2015, ApJ, 812, 49

Hopkins P. F., 2013, MNRAS, 428, 2840

Intema H. T., Jagannathan P., Mooley K. P., Frail D. A., 2016, ArXiv e-prints [astro-ph:1603.04368]

Kale R., Dwarakanath K. S., 2012, ApJ, 744, 46

Kale R., Venturi T., Giacintucci S., Dallacasa D., Cassano
R., Brunetti G., Cuciti V., Macario G., Athreya R., 2015, A\&A, 579, A92

Kang H., Ryu D., 2013, ApJ, 764, 95

Kang H., Ryu D., 2015, ApJ, 809, 186

Kang H., Ryu D., 2016, ArXiv e-prints [astro-ph:1602.03278]

Kang H., Ryu D., Jones T. W., 2012, ApJ, 756, 97

Kempner J. C., Blanton E. L., Clarke T. E., Enßlin T. A., Johnston-Hollitt M., Rudnick L., 2004, in T. Reiprich, J. Kempner, \& N. Soker ed., The Riddle of Cooling Flows in Galaxies and Clusters of galaxies Conference Note: A Taxonomy of Extended Radio Sources in Clusters of Galaxies. pp 335-+

Kempner J. C., Sarazin C. L., 2001, ApJ, 548, 639

Klypin A., Kravtsov A. V., Bullock J. S., Primack J. R., 2001, ApJ, 554, 903

Knollmann S. R., Knebe A., 2009, ApJS, 182, 608

Komissarov S. S., Gubanov A. G., 1994, A\&A, 285

Landau L. D., Lifshitz E. M., 1959, Fluid mechanics

Lindner R. R., Baker A. J., Hughes J. P., Battaglia N., Gupta N., Knowles K., Marriage T. A., Menanteau F., Moodley K., Reese E. D., Srianand R., 2014, ApJ, 786, 49

Macario G., Markevitch M., Giacintucci S., Brunetti G., Venturi T., Murray S. S., 2011, ApJ, 728, 82

Middelberg E., Norris R. P., Cornwell T. J., Voronkov M. A., Siana B. D., Boyle B. J., Ciliegi P., Jackson C. A., Huynh M. T., Berta S., Rubele S., Lonsdale C. J., Ivison R. J., Smail I., 2008, AJ, 135, 1276

Miniati F., 2014, ApJ, 782, 21

Miniati F., Ryu D., Kang H., Jones T. W., Cen R., Ostriker J. P., 2000, ApJ, 542, 608

Nagai D., Kravtsov A. V., Vikhlinin A., 2007, ApJ, 668, 1 Nakazawa K., Sarazin C. L., Kawaharada M., Kitaguchi T., Okuyama S., Makishima K., Kawano N., Fukazawa Y., Inoue S., Takizawa M., Wik D. R., Finoguenov A., Clarke T. E., 2009, PASJ, 61, 339

Norris R. P., Hopkins A. M., Afonso J., Brown S., Condon J. J., Dunne L., Feain I., Hollow R., et al. 2011, PASA, 28,215

Nuza S. E., Hoeft M., van Weeren R. J., Gottlöber S., Yepes G., 2012, MNRAS, 420, 2006

Ogrean G. A., Brüggen M., van Weeren R. J., Burgmeier A., Simionescu A., 2014, MNRAS, 443, 2463

Ogrean G. A., Brüggen M., van Weeren R. J., Röttgering H., Croston J. H., Hoeft M., 2013, MNRAS, 433, 812

Pandey-Pommier M., Richard J., Combes F., Dwarakanath K. S., Guiderdoni B., Ferrari C., Sirothia S., Narasimha D., 2013, A\&A, 557, A117

Pfrommer C., Springel V., Enßlin T. A., Jubelgas M., 2006, MNRAS, 367, 113

Pinzke A., Oh S. P., Pfrommer C., 2013, MNRAS, 435, 1061

Pizzo R. F., de Bruyn A. G., Feretti L., Govoni F., 2008, A\&A, 481, L91

Planck Collaboration Ade P. A. R., Aghanim N., ArmitageCaplan C., Arnaud M., Ashdown M., Atrio-Barandela F., Aumont J., Aussel H., Baccigalupi C., et al. 2015, A\&A, 581, A14

Prada F., Klypin A. A., Cuesta A. J., Betancort-Rijo J. E., Primack J., 2012, MNRAS, 423, 3018

Randall S. W., Clarke T. E., Nulsen P. E. J., Owers M. S., 
Sarazin C. L., Forman W. R., Murray S. S., 2010, ApJ, 722,825

Randall S. W., Clarke T. E., van Weeren R. J., Intema H. T., Dawson W. A., Mroczkowski T., Blanton E. L., Bulbul E., Giacintucci S., 2016, ArXiv e-prints [astro-ph:1604.03551]

Rengelink R. B., Tang Y., de Bruyn A. G., Miley G. K., Bremer M. N., Roettgering H. J. A., Bremer M. A. R., 1997, A\&A S, 124

Reynolds S. P., 2008, ARA\&A, 46, 89

Riseley C. J., Scaife A. M. M., Wise M. W., Clarke A. O., 2016, ArXiv e-prints [astro-ph:1611.01273]

Rottgering H. J. A., Wieringa M. H., Hunstead R. W., Ekers R. D., 1997, MNRAS, 290, 577

Ryu D., Kang H., Hallman E., Jones T. W., 2003, ApJ, 593, 599

Sarazin C., Hogge T., Chatzikos M., Wik D., Giacintucci S., Clarke T., Wong K., Gitti M., Finoguenov A., 2014, in The X-ray Universe 2014 XMM-Newton and Chandra Observations of the Remarkable Dynamics of the Intracluster Medium and Radio Sources in the Clusters Abell 2061 and 3667. p. 181

Schaal K., Springel V., Pakmor R., Pfrommer C., Nelson D., Vogelsberger M., Genel S., Pillepich A., Sijacki D., Hernquist L., 2016, ArXiv e-prints [astro-ph:1604.07401]

Sembolini F., Elahi P. J., Pearce F. R., Power C., Knebe A., Kay S. T., Cui W., Yepes G., et al. 2016, MNRAS, 459, 2973

Sembolini F., Yepes G., De Petris M., Gottlöber S., Lamagna L., Comis B., 2013, MNRAS, 429, 323

Shakouri S., Johnston-Hollitt M., Pratt G. W., 2016, MNRAS, 459, 2525

Shimwell T. W., Markevitch M., Brown S., Feretti L., Gaensler B. M., Johnston-Hollitt M., Lage C., Srinivasan R., 2015, MNRAS, 449, 1486

Sifón C., Menanteau F., Hughes J. P., Carrasco M., Barrientos L. F., 2014, A\&A, 562, A43

Skillman S. W., Hallman E. J., O'Shea B. W., Burns J. O., Smith B. D., Turk M. J., 2011, ApJ, 735, 96

Skillman S. W., O'Shea B. W., Hallman E. J., Burns J. O., Norman M. L., 2008, ApJ, 689, 1063

Skillman S. W., Xu H., Hallman E. J., O'Shea B. W., Burns J. O., Li H., Collins D. C., Norman M. L., 2013, ApJ, 765, 21

Slee O. B., Roy A. L., Murgia M., Andernach H., Ehle M., 2001, AJ, 122, 1172

Springel V., 2005, MNRAS, 364, 1105

Stasyszyn F., Nuza S. E., Dolag K., Beck R., Donnert J., 2010, MNRAS, 408, 684

Stobie R. S., 1980, Journal of the British Interplanetary Society, 33, 323

Stroe A., Shimwell T., Rumsey C., van Weeren R., Kierdorf M., Donnert J., Jones T. W., Röttgering H. J. A., Hoeft M., Rodríguez-Gonzálvez C., Harwood J. J., Saunders R. D. E., 2016, MNRAS, 455, 2402

Stroe A., van Weeren R. J., Intema H. T., Röttgering H. J. A., Brüggen M., Hoeft M., 2013, A\&A, 555, A110

Subrahmanyan R., Beasley A. J., Goss W. M., Golap K., Hunstead R. W., 2003, AJ, 125, 1095

van Weeren R. J., Brüggen M., Röttgering H. J. A., Hoeft M., Nuza S. E., Intema H. T., 2011, A\&A, 533, A35 van Weeren R. J., Brunetti G., Brüggen M., AndradeSantos F., Ogrean G. A., Williams W. L., Röttgering H. J. A., Dawson e., 2016, ApJ, 818, 204

van Weeren R. J., Fogarty K., Jones C., Forman W. R., Clarke T. E., Brüggen M., Kraft R. P., Lal D. V., Murray S. S., Röttgering H. J. A., 2013, ApJ, 769, 101

van Weeren R. J., Hoeft M., Röttgering H. J. A., Brüggen M., Intema H. T., van Velzen S., 2011, A\&A, 528, A38+ van Weeren R. J., Röttgering H. J. A., Brüggen M., 2011, A\&A, 527, A114+

van Weeren R. J., Röttgering H. J. A., Brüggen M., Cohen A., 2009a, A\&A, 508, 75

van Weeren R. J., Röttgering H. J. A., Brüggen M., Cohen A., 2009b, A\&A, 505, 991

van Weeren R. J., Röttgering H. J. A., Brüggen M., Hoeft M., 2010, Science, 330, 347

van Weeren R. J., Röttgering H. J. A., Intema H. T., Rudnick L., Brüggen M., Hoeft M., Oonk J. B. R., 2012, A\&A, 546, A124

Vazza F., Brüggen M., 2014, MNRAS, 437, 2291

Vazza F., Brüggen M., van Weeren R., Bonafede A., Dolag K., Brunetti G., 2012, MNRAS, 421, 1868

Vazza F., Brüggen M., Wittor D., Gheller C., Eckert D., Stubbe M., 2016, MNRAS, 459, 70

Vazza F., Brunetti G., Gheller C., 2009, MNRAS, 395, 1333

Vazza F., Dolag K., Ryu D., Brunetti G., Gheller C., Kang H., Pfrommer C., 2011, MNRAS, 418, 960

Venturi T., Giacintucci S., Dallacasa D., Cassano R., Brunetti G., Macario G., Athreya R., 2013, A\&A, 551, A24

Wenger M., Ochsenbein F., Egret D., Dubois P., Bonnarel F., Borde S., Genova F., Jasniewicz G., Laloë S., Lesteven S., Monier R., 2000, A\&A S, 143, 9

Yuan Z. S., Han J. L., Wen Z. L., 2015, ApJ, 813, 77 